\title{
ON THE $\ell$-ADIC COHOMOLOGY OF SOME $p$-ADICALLY UNIFORMIZED SHIMURA VARIETIES
}

\author{
$\mathrm{XU}$ SHEN† \\ Fakultät für Mathematik, Universität Regensburg, Universitaetsstr. 31, \\ 93040 Regensburg, Germany \\ (xu.shen@mathematik.uni-regensburg.de)
}

(Received 12 December 2014; revised 2 November 2016; accepted 11 November 2016;

first published online 1 December 2016)

\begin{abstract}
We determine the Galois representations inside the $\ell$-adic cohomology of some unitary Shimura varieties at split places where they admit uniformization by finite products of Drinfeld upper half spaces. Our main results confirm Langlands-Kottwitz's description of the cohomology of Shimura varieties in new cases.
\end{abstract}

Keywords: Shimura varieties; $p$-adic uniformization; $\ell$-adic cohomology

2010 Mathematics subject classification: Primary $11 \mathrm{G} 18$

Secondary 14G35

\section{Introduction}

The aim of this article is to determine the Galois representations inside the $\ell$-adic cohomology of some unitary Shimura varieties at split places where they admit uniformization by finite products of Drinfeld upper half spaces ([34, Theorem 6.50] and [44]). The main results confirm Langlands-Kottwitz's description of the cohomology of Shimura varieties in new cases.

For the Shimura varieties with good reductions at $p(\neq \ell)$, Langlands and Kottwitz have given a conjectural description of the Galois representations inside the cohomology (cf. [23]). Roughly it says that, the Galois representation associated to an automorphic representation when restricting to a place above $p$ is given by the local Langlands correspondence for the local reductive group. To prove such a result, Langlands's idea is to analyze the cohomology of Shimura varieties by computing the alternating sum of the traces of Hecke operators twisted by a Frobenius correspondence on the cohomology. By Lefschetz trace formula, this needs to understand the set of points on Shimura varieties 
over finite fields. Kottwitz introduced some group theoretic triples $\left(\gamma_{0} ; \gamma, \delta\right)$ in $[25]$ which, roughly speaking, parameterize isogeny classes of points of Shimura varieties over finite fields. There he also proved a formula for the traces of Hecke operators twisted by a Frobenius by using these triples. Then to get the desired description, one should stabilize this formula and compare it with the Arthur-Selberg trace formula (see [23]). In some cases this description has been proved, for example see [24, 30]. Here in our case, even for the maximal level at $p$, these varieties have bad reductions. And in fact we also want to treat the cases of arbitrary levels at $p$.

In [39] Scholze has developed the Langlands-Kottwitz approach for some PEL Shimura varieties with arbitrary level at $p$. There the local hypothesis are made to ensure the local reductive groups are quasi-split, so that there is no problem for the definition of Kottwitz triples. Also, Scholze proved that the set of all equivalent effective Kottwitz triples can parameterize all the fixed points. The key new ingredient is to define some test functions by deformation spaces of $p$-divisible groups with some additional structures. This approach avoids the study of local models of Shimura varieties with bad reductions, and gives less information about these functions. However, the definition of these test functions is conceptually elegant, and sufficient for applications in many ways. Scholze then studied the properties of these functions and proved a formula similar to the one of Kottwitz in [25]. Using this formula Scholze and Shin in [40] have proved some character identities about the transfers of the test functions defined in [39], and deduce many results about the cohomology of some compact unitary Shimura varieties for arbitrary level at $p$, which confirm the expected descriptions of Langlands-Kottwitz. Note to have such a description one needs to know the local Langlands correspondence for the related reductive groups. In [40] their assumptions are made such that the local reductive groups at $p$ are products of Weil restriction of general linear groups.

For the PEL Shimura varieties with reductive groups $G$ non-quasi-split at $p$, we also want to describe their points modulo $p$ and their $\ell$-adic cohomology. However, there are some group theoretic problems due to the non-quasi-split property. Namely, in this set-up the set of equivalent Kottwitz triples (in the usual sense) will not be enough to parameterize all the points valued in a finite field $\mathbb{F}_{p^{t}}$. We can indeed find the pairs $(\gamma, \delta)$ associated to each isogeny class over $\mathbb{F}_{p^{t}}$. But the conjugacy class of the norm $N \delta$ does not always contain an element of $G\left(\mathbb{Q}_{p}\right)$ (cf. [21]). This is an obstruction to find a $\gamma_{0} \in G(\mathbb{Q})$ such that $\left(\gamma_{0} ; \gamma, \delta\right)$ forms a Kottwitz triple. Nevertheless, we can introduce some reasonable test functions $\phi_{\tau, h}$ at $p$ in the same way as [39] whose twisted orbital integrals contribute to the trace formula. This was already noted by Scholze in [39]. Following [32, Conjecture 5.7] and [33, Conjecture 10.2], one conjectures that if the conjugacy class of the norm $N \delta$ does not contain an element of $G\left(\mathbb{Q}_{p}\right)$, then the twisted orbital integral vanishes

$$
T O_{\delta \sigma}\left(\phi_{\tau, h}\right)=0
$$

This is certainly a new phenomenon in the non-quasi-split case. If one can prove this result, then only the points parameterized by the Kottwitz triples contribute to the trace formula, and one has a similar formula as [23, 39]. Going through further, one can continue the process of stabilization or pseudostabilization to compute the cohomology. To get the desired description of the cohomology, one still needs to know the local Langlands 
correspondence for $G\left(\mathbb{Q}_{p}\right)$ and a suitable character identity for the twisted transfer of $\phi_{\tau, h}$ (cf. [40, Conjecture 7.1], which is a weaker form of [14, Conjectures 6.1.1 and 6.2.3]).

This paper deals with a special example where we can prove the above two main points for the test functions $\phi_{\tau, h}$. Also, for this case at hand, the local Langlands correspondence has been known. So we can get desired description of the cohomology. Let $S h_{K}$ be a Shimura variety over its local reflex field $E$ with the open compact subgroup in the form $K=K^{p} K_{p} \subset G\left(\mathbb{A}_{f}\right)$, such that at $p$ it admits uniformization by $r$ products of Drinfeld moduli spaces $\mathcal{M}_{D r, K_{p}}$ with level $K_{p}$. Let $\xi$ be an irreducible algebraic representation of $G$ over $\overline{\mathbb{Q}}_{\ell}$. By a standard construction, we have a $\overline{\mathbb{Q}}_{\ell}$-local system $\mathcal{L}_{\xi}$ on each Shimura variety $S h_{K}$ for $K \subset G\left(\mathbb{A}_{f}\right)$. We are interested in the virtual $G\left(\mathbb{A}_{f}\right) \times W_{E}$-representation defined by the alternating sum of $\ell$-adic cohomology

$$
H_{\xi}=\sum_{i}(-1)^{i} \underset{K}{\lim _{K}} H^{i}\left(S h_{K} \times \overline{\mathbb{Q}}_{p}, \mathcal{L}_{\xi}\right)
$$

where $K$ runs over the set of open compact subgroups of $G\left(\mathbb{A}_{f}\right)$. The local reductive group $G_{\mathbb{Q}_{p}}$ is a product of some inner forms of $G L_{n}$ (together with $\mathbb{G}_{m}$ ), so the local Langlands correspondence has been known (cf. $[2,19]$ ). For any smooth irreducible representation $\pi_{p}$ of $G\left(\mathbb{Q}_{p}\right)$, let $\varphi_{\pi_{p}}$ be the associated local Langlands parameter. Recall associated to the Shimura data $\left(G, h^{-1}\right)$ we have the representation $r_{-\mu}$ of the Langlands dual group ${ }^{L}\left(G_{E}\right)$ introduced in [22, Lemma 2.1.2]. The main theorem is as follows.

Theorem 1.1. With the notations as above, we have an identity

$$
H_{\xi}=\sum_{\pi_{f}} a\left(\pi_{f}\right) \pi_{f} \otimes\left(r_{-\mu} \circ \varphi_{\pi_{p}} \mid W_{E}\right)|-|^{r(1-n) / 2}
$$

as virtual $G\left(\mathbb{Z}_{p}\right) \times G\left(\mathbb{A}_{f}^{p}\right) \times W_{E}$-representations. Here $\pi_{f}$ runs through irreducible admissible representations of $G\left(\mathbb{A}_{f}\right)$, the integer $a\left(\pi_{f}\right)$ is as in [24, p. 657].

We first prove the theorem for case $r=1$ by using the results of Boyer [6] and Dat [8], thus avoiding the counting points method for these Shimura varieties. In fact, the global $p$-adic uniformization leads to a Hochschild-Serre spectral sequence for the $\ell$-adic cohomology of these Shimura varieties, from which we get a formula (in [12] Fargues called it as a $p$-adic Matsushima formula) for $H_{\xi}$. Then using Boyer's description of the cohomology of Lubin-Tate spaces, the Faltings-Fargues isomorphism for the towers of Lubin-Tate and Drinfeld, and Dat's results about extension of elliptic representations, we get the desired formula in the theorem. Here we prove the identity as $G\left(\mathbb{A}_{f}\right) \times W_{E}$-representations. We note that essentially the same idea had already appeared in $[8, \S 5]$.

To prove the result in general case, we develop the theory of test functions by means of deformation spaces of $p$-divisible groups in our context as in [39]. We also adapt some notations from [38]. To prove the vanishing results and character identities for these test functions, we use the formula for $H_{\xi}$ in the case $r=1$ proved previously. Thus these are some global arguments. We note that in [32] Rapoport conjectured the vanishing results for the example there with maximal level at $p$, and by some explicit combinatorial description of the test function Waldspurger proved this conjecture in the 
case $r=1$. There the test functions were constructed by the theory of local models which describes the bad reduction of the Shimura varieties. These functions satisfy the required character identities. So in this case $r=1$ and the level structure $K_{p}$ at $p$ is maximal, the result was more or less known in [32]. In fact in [32] Rapoport restricted on the trivial coefficients and concentrated on the local semisimple zeta functions (see the below corollary).

In [41] we shall use our results for the test functions to describe the cohomology of quaternionic and related unitary Shimura varieties at ramified places (see $\S 7$ for more details).

In a series of papers [15-17], Harris had studied the supercuspidal part of the cohomology. At that time, one did not know the local Galois representations inside the cohomology are given by the local Langlands correspondence, except for the cases $n<$ $p$ [16]. In fact, in these papers Harris tried to prove the local Langlands correspondence for $G L_{n}$ by studying the supercuspidal part of the cohomology of these Shimura varieties. Later, in [18] Harris and Taylor successfully achieved this by studying the cohomology of another class of Shimura varieties. We note that the same result as in the above theorem for Harris-Taylor's Shimura varieties was proved in [37] implicitly.

From this theorem we get the following corollary concerning the local semisimple zeta functions of our Shimura varieties. Let $\widetilde{E}$ be the global reflex field, and $v$ be a place of $\widetilde{E}$ above $p$ such that $E=\widetilde{E}_{v}$.

Corollary 1.2. In the situation of the theorem, let $K \subset G\left(\mathbb{A}_{f}\right)$ be any sufficiently small open compact subgroup. Then the semisimple local Hasse-Weil zeta function of $S h_{K}$ at the place $v$ of $\widetilde{E}$ is given by

$$
\zeta_{v}^{s s}\left(S h_{K}, s\right)=\prod_{\pi_{f}} L^{s s}\left(s-r(n-1) / 2, \pi_{p}, r_{-\mu}\right)^{a\left(\pi_{f}\right) \operatorname{dim} \pi_{f}^{K}} .
$$

In the case $r=1$ Dat has proved the Weight-Monodromy conjecture for these Shimura varieties (cf. [8, 5.2]). Then by $[32, \S 2]$ one can recover the classical Hasse-Weil zeta function.

Corollary 1.3. Let $r=1$ and $K \subset G\left(\mathbb{A}_{f}\right)$ be any sufficiently small open compact subgroup in the situation of the theorem. Then the local Hasse-Weil zeta function of $S h_{K}$ at the place $v$ of $\widetilde{E}$ is given by

$$
\zeta_{\nu}\left(S h_{K}, s\right)=\prod_{\pi_{f}} L\left(s-r(n-1) / 2, \pi_{p}, r_{-\mu}\right)^{a\left(\pi_{f}\right) \operatorname{dim} \pi_{f}^{K}} .
$$

In [20] Ito proved the Weight-Monodromy conjecture for the varieties which are $p$-adically uniformized by the maximal level Drinfeld upper half spaces. In [20, Theorem 6.2] an application to the local zeta function was also presented. These varieties are the Galois twisted versions of the connected components of our Shimura varieties studied here with $r=1$ and $K_{p}$ maximal (see [34, Theorem 6.50] and [44, 2.13]).

Shortly after the first version of this paper, Mieda claimed that the Weight-Monodromy conjecture holds true for the general $p$-adically uniformized Shimura varieties studied 
here, i.e., $r$ is not necessary 1 , and one can argue as in $\S 3$ to prove the above Theorem 1.1 (cf. [28]). In particular in Corollary $1.3 r$ can be an arbitrary positive integer. However, as the reader can see in this introduction, our general purpose is trying to prove results for as many cases as possible. Our theory of test functions developed in $\S \S 4-6$ by Scholze's method, will be used in [41] in an essential way to prove results for some other Shimura varieties.

We give an overview of the content of this article. In $\S 2$ we introduce the $p$-adically uniformized Shimura varieties which we are interested in. In $\S 3$ we deduce the cohomology of the Shimura varieties uniformized by one Drinfeld moduli spaces by using the results of Boyer and Dat. Then in $\S 4$ we define the test functions $\phi_{\tau, h}$ by Scholze's method, and list their properties. Here we just state the facts since the arguments and proofs are the same as those in [39]. In $\S 5$ we prove the vanishing property of these test functions by global method, and establish the trace formula as a sum over the set of equivalent Kottwitz triples. In $\S 6$ we use the formula for $H_{\xi}$ in the case $r=1$ proved in $\S 3$ to deduce the character identity of the transfers $f_{\tau, h}$ of $\phi_{\tau, h}$. Finally in $\S 7$ we deduce the theorem for the general case. Corollaries for the local (semisimple) zeta functions of Shimura varieties are stated.

\section{Some $p$-adically uniformized Shimura varieties}

We now introduce some unitary Shimura varieties which admit $p$-adic uniformization by finite products of Drinfeld upper half spaces. They were first introduced by Rapoport-Zink [34] and Varshavsky [43, 44] as higher-dimensional generalization of the Cherednik's theorem for Shimura curves as presented in $[4,5,10]$. We note that some special higher-dimensional cases already appeared in [32].

Let $p$ be a prime number. Fix an imaginary quadratic field $K$ in which $p$ splits. The two primes of $K$ above $p$ will be denoted by $u$ and $u^{c}$, and the complex conjugation of $\operatorname{Gal}(K / \mathbb{Q})$ will be denoted by $c$. Let $F^{+} \mid \mathbb{Q}$ be a totally real field of degree $N$. Set $F=$ $F^{+} K$, so that $F$ is a CM-field with maximal totally real subfield $F^{+}$. Let $\varpi_{1}, \varpi_{2}, \ldots, \varpi_{s}$ denote the primes of $F$ above $u$, and let $v_{1}, v_{2}, \ldots, v_{s}$ denote their restrictions to $F^{+}$. Fix an integer $1 \leqslant r \leqslant s$. Let $B / F$ denote a central division algebra of dimension $n^{2}$ over $F$ such that

- the opposite algebra $B^{o p}$ is isomorphic to $B \otimes_{K, c} K$;

- at any place $x$ of $F$ which is not split over $F^{+}, B_{x}$ is split (here and in the following $\left.B_{x}=B \otimes F_{x}\right)$

- at the places $\varpi_{1}, \ldots, \varpi_{r}, \varpi_{1}^{c}, \ldots, \varpi_{r}^{c}$ (for $1 \leqslant i \leqslant s, \varpi_{i}^{c}$ is the place over $u^{c}$ which induces also $v_{i}$ on $\left.F^{+}\right) B$ is ramified with invariants

$$
i n v_{\varpi_{i}} B=\frac{1}{n}, \quad i n v_{\varpi_{i}^{c}} B=-\frac{1}{n} ;
$$

- at the places $\varpi_{r+1}, \ldots, \varpi_{s}, \varpi_{r+1}^{c}, \ldots, \varpi_{s}^{c}$ the invariants of $B$ are arbitrary but satisfy

$$
i n v_{\varpi_{i}} B=-i n v_{\varpi_{i}^{c}} B .
$$

Note that we assume in particular $s=t$ by the notation of $[34,6.38]$. 
We assume that there is an involution of second kind $*$ on $B$. Moreover, we can choose some alternating pairing $\langle$,$\rangle on V \times V \rightarrow \mathbb{Q}$ for the $B \otimes_{F} B^{o p}$ module $V:=B$, which corresponds to another involution of second kind $\sharp$ on $B$. The associated reductive group $G / \mathbb{Q}$ is defined by

$$
G(R)=\left\{(g, \lambda) \in\left(B^{o p} \otimes_{\mathbb{Q}} R\right)^{\times} \times R^{\times} \mid g g^{\sharp}=\lambda\right\},
$$

for any $\mathbb{Q}$-algebra $R$. Let $G_{1}$ be the kernel of the map $G \rightarrow \mathbb{G}_{m},(g, \lambda) \mapsto \lambda$, which can be viewed as a group over $F^{+}$. For $1 \leqslant i \leqslant r$, choose distinguished embeddings $\tau_{i}: F^{+} \hookrightarrow \mathbb{R}$. As in $[34,6.40]$, we assume that we can make the choice of the alternating pairing on $V \times V \rightarrow \mathbb{Q}$ and the isomorphism $\iota: \overline{\mathbb{Q}}_{p} \simeq \mathbb{C}$ such that

- if $\sigma: F^{+} \hookrightarrow \mathbb{R}$ is an embedding, then $G_{1} \times{ }_{F^{+}, \sigma} \mathbb{R}$ is isomorphic to the unitary group $U(1, n-1)$ if $\sigma=\tau_{i}$ for $1 \leqslant i \leqslant r$ and $U(n)$ otherwise;

- under the bijection $\operatorname{Hom}\left(F^{+}, \mathbb{C}\right)=\operatorname{Hom}\left(F^{+}, \mathbb{R}\right) \rightarrow \operatorname{Hom}\left(F^{+}, \overline{\mathbb{Q}}_{p}\right)$ induced by $\iota$, $\tau_{1}, \ldots, \tau_{r}$ induce the primes $v_{1}, \ldots, v_{r}$ of $F^{+}$above $p$.

Let $B_{\varpi_{i}}^{\times}$be the local reductive group over $F_{\varpi_{i}}$ associated to the units in $B_{\varpi_{i}}$ for $1 \leqslant i \leqslant r$. Then under our assumptions we have

$$
G_{\mathbb{Q}_{p}} \simeq \operatorname{Res}_{F_{\varpi_{1}}} B_{\varpi_{1}}^{\times} \times \cdots \times \operatorname{Res}_{F_{\varpi_{r}}} B_{\varpi_{r}}^{\times} \times G_{D^{\prime}} \times \mathbb{G}_{m}
$$

with obvious definition of the factor $G_{D^{\prime}}$ which is associated to the semisimple algebra $D^{\prime}=\prod_{i=r+1}^{s} B_{\varpi_{i}}$ over $\mathbb{Q}_{p}$. Let $E$ be the composition of the fields $F_{\varpi_{1}}, \ldots, F_{\varpi_{r}}$, which will be our local reflex field.

As in $[34,6.37]$ we have a homomorphism $h: \operatorname{Res}_{\mathbb{C} \mid \mathbb{R}} \mathbb{G}_{m} \rightarrow G_{\mathbb{R}}$ which corresponds to our signature condition. Then the datum $\left(G, h^{-1}\right)$ defines a projective Shimura variety $S h_{K}$ over $E$ for any compact open subgroup $K \subset G\left(\mathbb{A}_{f}\right)$ (cf. [25]). The conjugacy class of the cocharacter $\mu: \mathbb{G}_{m} \longrightarrow G_{\overline{\mathbb{Q}}_{p}}$ associated to $h$ is defined over $E$ (cf. [34, 6.40]). For sufficiently small open compact subgroup $K^{p} \subset G\left(\mathbb{A}_{f}^{p}\right)$, we have a projective scheme $S_{K^{p}}$ over $O_{E}$ (the integer ring of $E$ ) which is a moduli space of some abelian varieties with additional structures. This moduli space is defined in a similar way as those introduced in [25], but contrary to the later case, it is not smooth. For any locally noetherian scheme $S$ over $O_{E}, S_{K^{p}}(S)$ is the isomorphism classes of quadruples $\left(A, \lambda, \iota, \bar{\eta}^{p}\right)$ consisting of

- a projective abelian scheme $A$ over $S$ up to prime-to- $p$ isogeny;

- a polarization $\lambda: A \rightarrow A^{D}$ of degree prime to $p$ (here and in the following, the upper subscript $D$ means the Cartier dual);

- a homomorphism $\iota: O_{B} \rightarrow \operatorname{End}(A)$ satisfying the determinant condition and compatible with $\lambda$;

- a level structure $\bar{\eta}^{p}$ of type $K^{p}$.

For more details we refer to [34, Definition 6.9]. As usual on generic fibers we have the isomorphism

$$
S_{K^{p}} \times O_{E} E \simeq \coprod_{k e r^{1}(\mathbb{Q}, G)} S h_{K_{p}^{0} K^{p}},
$$

where $K_{p}^{0} \subset G\left(\mathbb{Q}_{p}\right)$ is the maximal open compact subgroup $O_{B_{\varpi_{1}}}^{\times} \times \cdots \times O_{B_{\varpi_{r}}}^{\times} \times O_{D^{\prime}}^{\times} \times$ $\mathbb{Z}_{p}^{\times}$ 
We set $D_{i}:=B_{\varpi_{i}}$ from now on. For any locally noetherian scheme $S$ over $O_{E}$ on which $p$ is locally nilpotent, let $A / S$ be an abelian scheme coming from an $S$-valued point of $S_{K^{p}}$. Looking at its $p$-divisible group, we get a decomposition

$$
A\left[p^{\infty}\right]=\left(H_{1} \oplus \cdots \oplus H_{r} \oplus H^{\prime}\right) \oplus\left(H_{1} \oplus \cdots \oplus H_{r} \oplus H^{\prime}\right)^{D},
$$

where for $1 \leqslant i \leqslant r, H_{i}$ is a $\varpi_{i}$-divisible $O_{D_{i}}$-module, $H^{\prime}$ is a $D^{\prime}$-group in the sense of $\left[38\right.$, Definition 4.1] which is the sum of the étale $\varpi_{i}$-divisible $O_{D_{i}}$-modules for $i=$ $r+1, \ldots, s$. In particular, after fixing a point in the special fiber of $S_{K} p$ we can consider the associated (formal) Rapoport-Zink space $\widehat{\mathcal{M}}$, which has the decomposition (cf. [34, Proposition 6.49])

$$
\begin{aligned}
\widehat{\mathcal{M}} & \simeq \widehat{\mathcal{M}}_{D r, \varpi_{1}} \times \cdots \times \widehat{\mathcal{M}}_{D r, \varpi_{r}} \times G_{D^{\prime}}\left(\mathbb{Q}_{p}\right) / O_{D^{\prime}}^{\times} \times \mathbb{Q}_{p}^{\times} / \mathbb{Z}_{p}^{\times} \\
& \simeq\left(\prod_{i=1}^{r} \widehat{\Omega}_{F_{\varpi_{i}}}^{n} \times S p f O_{\check{E}} \times D_{i}^{\times} / K_{p, \varpi_{i}}^{0}\right) \times G_{D^{\prime}}\left(\mathbb{Q}_{p}\right) / O_{D^{\prime}}^{\times} \times \mathbb{Q}_{p}^{\times} / \mathbb{Z}_{p}^{\times} \\
& \simeq\left(\prod_{i=1}^{r} \widehat{\Omega}_{F_{\varpi_{i}}}^{n} \times \operatorname{SpfO_{\check {E}}}\right) \times G\left(\mathbb{Q}_{p}\right) / K_{p}^{0},
\end{aligned}
$$

where for $i=1, \ldots, r, \widehat{\mathcal{M}}_{D r, \varpi_{i}}$ is the formal Drinfeld moduli space associated to the local data, $K_{p, \varpi_{i}}^{0} \subset D_{i}^{\times}$is the maximal open compact subgroup and $\widehat{\Omega}_{F_{\varpi_{i}}}^{n}$ is the formal Drinfeld upper half space over $\operatorname{SpfO}_{F_{\varpi_{i}}}$. Here $\check{E}$ is the completion of the maximal unramified extension of $E$. The associated reductive group $J_{b}$ has the form (cf. [34, 6.44 p. 310, 6.46 and 6.49])

$$
J_{b} \simeq \operatorname{Res}_{F_{\bar{D}_{1}}} G L_{n} \times \cdots \times \operatorname{Res}_{F_{\bar{\sigma}_{r}}} G L_{n} \times G_{D^{\prime}} \times \mathbb{G}_{m} .
$$

Now we have the following theorem which says that our Shimura varieties admit global p-adic uniformization.

Theorem 2.1 [34, Theorem 6.50]. As $K^{p}$ varies, there is a $G\left(\mathbb{A}_{f}^{p}\right)$-equivariant isomorphism of formal schemes

$$
\coprod_{\operatorname{ker}^{1}(\mathbb{Q}, G)} I(\mathbb{Q}) \backslash\left(\prod_{i=1}^{r} \widehat{\Omega}_{F_{\varpi_{i}}}^{n} \times S p f O_{\check{E}}\right) \times G\left(\mathbb{A}_{f}\right) / K \simeq \widehat{S}_{K^{p}} \times S p f O_{\check{E}},
$$

where $K=K_{p}^{0} K^{p}$ and $\widehat{S}_{K^{p}}$ is the formal completion of $S_{K^{p}}$ along its special fiber. The group $I$ is an inner form of $G$ over $\mathbb{Q}$ such that $I\left(\mathbb{Q}_{p}\right)=J_{b}\left(\mathbb{Q}_{p}\right), I\left(\mathbb{A}_{f}^{p}\right)=G\left(\mathbb{A}_{f}^{p}\right)$. This defines the action of $I(\mathbb{Q})$ used in forming the quotient above. The natural descent datum on the right hand side induces on the left hand side the natural descent datum on the first $r$ factors (under the above decomposition for $\widehat{\mathcal{M}}$ ) multiplied with an action of some explicit element $g \in G\left(\mathbb{Q}_{p}\right)$ on $G\left(\mathbb{A}_{f}\right) / K$.

Passing to rigid analytic fibers, we get a rigid analytic uniformization of these Shimura varieties $S h_{K_{p}^{0} K_{p}}$. Moreover, in the rigid analytic setting, we have the uniformization for arbitrary levels at $p$. More precisely, let $m \geqslant 1$ be an integer, we consider open compact subgroups of the form

$$
K_{p}^{m}=\left(1+\Pi_{1}^{m} O_{D_{1}}\right) \times \cdots \times\left(1+\Pi_{r}^{m} O_{D_{r}}\right) \times\left(1+p^{m} O_{D^{\prime}}\right) \times\left(1+p^{m} \mathbb{Z}_{p}\right) \subset G\left(\mathbb{Q}_{p}\right),
$$


where $\Pi_{i} \in D_{i}$ is a fixed uniformizer for each $1 \leqslant i \leqslant r$. Then we have the following $G\left(\mathbb{A}_{f}\right)$-equivariant isomorphism of rigid analytic spaces

$$
\begin{aligned}
I(\mathbb{Q}) & \backslash\left(\prod_{i=1}^{r} \mathcal{M}_{D r, \varpi_{i}, m}\right) \times G_{D^{\prime}}\left(\mathbb{Q}_{p}\right) /\left(1+p^{m} O_{D^{\prime}}\right) \times \mathbb{Q}_{p}^{\times} /\left(1+p^{m} \mathbb{Z}_{p}\right) \times G\left(\mathbb{A}_{f}^{p}\right) / K^{p} \\
\simeq & S h_{K_{p}^{m} K^{p}}^{r i g} \times \check{E},
\end{aligned}
$$

where $\mathcal{M}_{D r, \varpi_{i}, m}$ is the level $m$ rigid analytic Drinfeld moduli space for each $1 \leqslant i \leqslant r$. The above isomorphism is compatible with the two descent datums as in the above theorem on both sides.

\section{The cohomology of Shimura varieties I}

In this section we will assume $r=s=1$ and compute the $\ell$-adic cohomology of these $p$-adically uniformized Shimura varieties. The main ingredients are the Hochschild-Serre spectral sequences [12, 15], Boyer's description of the $\ell$-adic cohomology of the Lubin-Tate tower [6], the Fargues-Faltings isomorphism for the towers of Lubin-Tate and Drinfeld [11, 13], and Dat's results for extensions of elliptic representations [8]. We note that in the previous works [15-17], Harris had studied the supercuspidal part of the cohomology. In $[15,17]$ he did not prove that the associated local Galois representations are given by the local Langlands correspondences. In [16] he could prove this key fact for some special case $n<p$. In fact, Harris just constructed the local Langlands correspondences in these cases by the cohomology of these $p$-adically uniformized Shimura varieties. Later, in [18] Harris-Taylor studied the supercuspidal part of the $\ell$-adic cohomology of some other simple Shimura varieties to construct the local Langlands correspondence for $G L_{n}$ in the general case.

Fix a prime $l \neq p$. In this paragraph $r$ and $s$ are not necessary 1. Let $\xi$ be an irreducible representation of $G$ over $\overline{\mathbb{Q}}_{l}$. By standard construction, we have a $\overline{\mathbb{Q}}_{l}$-local system $\mathcal{L}_{\xi}$ on each Shimura variety $S h_{K}$ for $K \subset G\left(\mathbb{A}_{f}\right)$. We are interested in the virtual $G\left(\mathbb{A}_{f}\right) \times$ $W_{E}$-representation defined by the alternating sum of $\ell$-adic cohomology

$$
H_{\xi}=\sum_{i \geqslant 0}(-1)^{i} \underset{K}{\lim } H^{i}\left(S h_{K} \times \overline{\mathbb{Q}}_{p}, \mathcal{L}_{\xi}\right),
$$

where $K$ runs over the set of open compact subgroups of $G\left(\mathbb{A}_{f}\right)$.

Let the notations be as in the previous section with $r=s=1$. For $m \geqslant 1$, we have the rigid Rapoport-Zink space

$$
\mathcal{M}_{K_{p}^{m}}=\mathcal{M}_{D r, \varpi, m} \times \mathbb{Q}_{p}^{\times} /\left(1+p^{m} \mathbb{Z}_{p}\right) .
$$

Then the $p$-adic uniformization of $S h_{K_{p}^{m} K^{p}}$ gives rise to the following spectral sequence (cf. [15, Lemma 6], [12, Théorème 4.5.12]) see also [7, §4].

$$
\operatorname{Ext}_{J_{b}\left(\mathbb{Q}_{p}\right)}^{i}\left(H_{c}^{2(n-1)-j}\left(\mathcal{M}_{K_{p}^{m}} \times \mathbb{C}_{p}, \overline{\mathbb{Q}}_{l}(n-1)\right), \mathcal{A}(I)_{\xi}^{K^{p}}\right) \Rightarrow H^{i+j}\left(S h_{K_{p}^{m} K^{p}} \times \overline{\mathbb{Q}}_{p}, \mathcal{L}_{\xi}\right),
$$

where $\mathcal{A}(I)_{\xi}$ is the space of automorphic forms on $I$ such that each automorphic representation $\Pi \subset \mathcal{A}(I)_{\xi}$ has archimedean component $\Pi_{\infty}=\breve{\xi}$, the dual representation 
of $\xi ; \mathcal{A}(I)_{\xi}^{K^{p}}$ is the $K^{p}$-invariant subspace, and the Ext is taken in the category of smooth $\overline{\mathbb{Q}}_{l}$-representations of $J_{b}\left(\mathbb{Q}_{p}\right)$. For any $\Pi \subset \mathcal{A}(I)_{\xi}$, write its $p$-component as $\Pi_{p}$ and the restricted tensor product of its finite components outside $p$ as $\Pi^{p}$. Taking direct limits for levels on both sides and passing to the alternating sum, we get the equalities of virtual representations of $G\left(\mathbb{A}_{f}\right) \times W_{E}(\mathrm{cf} .[12$, Corollaire 4.6.3])

$$
\begin{aligned}
& H_{\xi}=\sum_{i, j \geqslant 0}(-1)^{i+j} \underset{m}{\lim _{m}} \operatorname{Ext}_{J_{b}\left(\mathbb{Q}_{p}\right)}^{i}\left(H_{c}^{j}\left(\mathcal{M}_{K_{p}^{m}} \times \mathbb{C}_{p}, \overline{\mathbb{Q}}_{l}(n-1)\right), \mathcal{A}(I)_{\xi}\right) \\
& =\sum_{\substack{i, j \geqslant 0 \\
\Pi \subset \mathcal{A}(I)_{\xi}}}(-1)^{i+j} \underset{m}{\underset{m}{\lim }} \operatorname{Ext}_{J_{b}\left(\mathbb{Q}_{p}\right)}^{i}\left(H_{c}^{j}\left(\mathcal{M}_{K_{p}^{m}} \times \mathbb{C}_{p}, \overline{\mathbb{Q}}_{l}(n-1)\right), \Pi_{p}\right) \otimes \Pi^{p} .
\end{aligned}
$$

This formula can also be viewed as an analogue of Mantovan's formula (cf. [27] and the last paragraph of this section). We would like to rewrite the above last formula in a slightly finer form. Recall that under our assumption $r=s=1$, the local reflex field $E=F_{\varpi}$ and the local reductive group has the form $G_{\mathbb{Q}_{p}}=\operatorname{Res}_{E \mid \mathbb{Q}_{p}} D^{\times} \times \mathbb{G}_{m}$. The cocharacter $\mu$ associated to the Shimura data factors as $\left(\mu_{0}, \mu_{1}\right)$ with $\mu_{0}$ (respectively $\left.\mu_{1}\right)$ the cocharacter of $\operatorname{Res}_{E \mid \mathbb{Q}_{p}} D^{\times}$(respectively $\mathbb{G}_{m}$ ). Recall associated to the cocharacter $\mu$ we have the representation $r_{-\mu}$ of the Langlands dual group ${ }^{L}\left(G_{E}\right)$ introduced in [22, Lemma 2.1.2]. We also have representations $r_{-\mu_{0}}$ and $r_{-\mu_{1}}$ associated to $\mu_{0}$ and $\mu_{1}$, respectively. An irreducible smooth representation $\Pi_{p}$ of $I\left(\mathbb{Q}_{p}\right)=J_{b}\left(\mathbb{Q}_{p}\right)$ decomposes as $\Pi_{p, 0} \otimes \chi_{p}$ where $\Pi_{p, 0}$ is an irreducible smooth representation of $G L_{n}(E)$ and $\chi_{p}$ is a character of $\mathbb{Q}_{p}^{\times}$.

Lemma 3.1. We have the equality

$$
\begin{aligned}
H_{\xi}= & \sum_{\substack{i, j \geqslant 0 \\
\Pi \subset \mathcal{A}(I)_{\xi}}}(-1)^{i+j} \underset{m}{\lim _{m}} \operatorname{Ext}_{G L_{n}(E)}^{i}\left(H_{c}^{j}\left(\mathcal{M}_{D r, m} \times \mathbb{C}_{p}, \overline{\mathbb{Q}}_{l}(n-1)\right), \Pi_{p, 0}\right) \\
& \left.\otimes r_{-\mu_{1}} \circ \varphi_{\chi_{p}}\right|_{W_{E}} \otimes \chi_{p} \otimes \Pi^{p},
\end{aligned}
$$

where $\varphi_{\chi_{p}}: W_{\mathbb{Q}_{p}} \longrightarrow{ }^{L}\left(\mathbb{G}_{m}\right)$ is the Langlands parameter associated to $\chi_{p}$.

Proof. Indeed, by the notation of [42]

$$
\operatorname{Mant}_{\mu}\left(\Pi_{p}\right):=\sum_{i, j \geqslant 0}(-1)^{i+j} \underset{m}{\lim _{m}} \operatorname{Ext}_{J_{b}\left(\mathbb{Q}_{p}\right)}^{i}\left(H_{c}^{j}\left(\mathcal{M}_{K_{p}^{m}} \times \mathbb{C}_{p}, \overline{\mathbb{Q}}_{l}(n-1)\right), \Pi_{p}\right),
$$

we have

$$
\operatorname{Mant}_{\mu}\left(\Pi_{p}\right)=\operatorname{Mant}_{\mu_{0}}\left(\Pi_{p, 0}\right) \otimes \operatorname{Mant}_{\mu_{1}}\left(\chi_{p}\right),
$$

with similar definitions of $\operatorname{Mant}_{\mu_{0}}\left(\Pi_{p, 0}\right)$ and $\operatorname{Mant}_{\mu_{1}}\left(\chi_{p}\right)$ using the corresponding Rapoport-Zink spaces. This equality comes from the decomposition of our Rapoport-Zink spaces $\mathcal{M}_{K_{p}^{m}}$. Now local class field theory tells us that

$$
\operatorname{Mant}_{\mu_{1}}\left(\chi_{p}\right)=\left.r_{-\mu_{1}} \circ \varphi_{\chi_{p}}\right|_{W_{E}} \otimes \chi_{p},
$$

which can also be viewed as a corollary of the results of [18] in the case $n=1$ (cf. [42, Proof of Theorem 7.5]). Now the lemma follows. 
We need to know the $\ell$-adic cohomology of the tower of Drinfeld spaces. To this end,

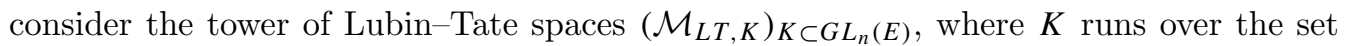
of open compact subgroups of $G L_{n}(E)$ contained in $G L_{n}\left(O_{E}\right)$. We have the following isomorphism.

Theorem $3.2[11,13]$. For each $i \geqslant 0$, there is a $G L_{n}(E) \times D^{\times} \times W_{E}$-equivariant isomorphism of groups

$$
\underset{K}{\lim } H_{c}^{i}\left(\mathcal{M}_{L T, K} \times \mathbb{C}_{p}, \overline{\mathbb{Q}}_{l}\right) \simeq \underset{m}{\lim } H_{c}^{i}\left(\mathcal{M}_{D r, m} \times \mathbb{C}_{p}, \overline{\mathbb{Q}}_{l}\right)
$$

In fact, for $0 \leqslant i \leqslant n-2$ we know the left hand side vanishes, so the right hand side also vanishes in these cases. We denote both sides by $H_{c}^{i}$. In [18] Harris-Taylor computed the supercuspidal part of the cohomology of Lubin-Tate spaces. Boyer in [6] has computed the remaining part. It involves elliptic representations of $G L_{n}(E)$. Recall an elliptic representation of $G L_{n}(E)$ is an irreducible smooth representation which has the same supercuspidal support as a discrete series representation. For more details we refer to $[8, \S 2]$. We state Boyer's results in the form as in $[8,4.1]$. For any irreducible smooth representation $\pi$ of $G L_{n}(E)$, let $\sigma(\pi)$ be its associated Weil-Deligne representation of $W_{E}$ by the local Langlands correspondence [18]. If $\pi$ is a discrete series representation, let $\sigma^{\prime}(\pi)$ be the unique irreducible sub Weil-Deligne representation of $\sigma(\pi)$. The set of irreducible smooth representations of $D^{\times}$will be denoted by $\operatorname{Irr}\left(D^{\times}\right)$, and an element of it will be usually denoted by $\rho$, with its contragredient denoted by $\rho^{\vee}$.

Theorem 3.3 [8, Théorème 4.1.2]. For $0 \leqslant i \leqslant n-1$, there is an isomorphism of $G L_{n}(E) \times D^{\times} \times W_{E}$-representations

$$
H_{c}^{n-1+i} \simeq \bigoplus_{\rho \in \operatorname{Irr}\left(D^{\times}\right)} \pi_{\rho}^{\leqslant i} \otimes \rho \otimes \sigma^{\prime}\left(J L(\rho)^{\vee}\right)|-|^{\frac{n / n_{\rho}-n}{2}-i+\frac{1-n}{2}},
$$

where $J L(\rho)$ is the discrete series representation of $G L_{n}(E)$ associated to $\rho$ by the Jacquet-Langlands correspondence, cf. [36]. $\pi_{\rho}|-|^{\frac{1-n_{\rho}}{2}} \otimes \cdots \otimes \pi_{\rho}|-|^{\frac{n_{\rho}-1}{2}}$ is its supercuspidal support, $n_{\rho}$ is an integer which divides $n$ such that $\pi_{\rho}$ is the (supercuspidal) representation of $G L_{n / n_{\rho}}(E), \pi_{\rho}^{\leqslant i}$ is the elliptic representation associated to $i$ with the same supercuspidal support as $\pi_{\rho}$ (cf. [8, 2.1.11 and 4.1.1]).

Here we have corrected the upper subscript of $|-|$ in $[6,8]$ according to $[18$, Theorem VII.1.5]. We note that $\sigma(J L(\rho))$ is the Weil-Deligne representation associated to $\rho \in \operatorname{Irr}\left(D^{\times}\right)$by the local Langlands correspondence for the inner form $D^{\times}$of $G L_{n}$, as expected naturally (cf. [2, 19]).

Combining Lemma 3.1 with Theorem 3.3, we can now prove the following theorem. Since the local reductive group $G_{\mathbb{Q}_{p}}$ is a product of inner forms of $G L_{n}$ (together with $\mathbb{G}_{m}$ ), the local Langlands correspondence for it is known $[2,19]$. For any smooth irreducible representation $\pi_{p}$ of $G\left(\mathbb{Q}_{p}\right)$, let $\varphi_{\pi_{p}}: W_{\mathbb{Q}_{p}} \longrightarrow{ }^{L}\left(G_{\mathbb{Q}_{p}}\right)$ be the associated local Langlands parameter. 
Theorem 3.4. With the notations as above, we have an identity

$$
H_{\xi}=\sum_{\pi_{f}} a\left(\pi_{f}\right) \pi_{f} \otimes\left(r_{-\mu} \circ \varphi_{\pi_{p}} \mid W_{E}\right)|-|^{(1-n) / 2}
$$

as virtual $G\left(\mathbb{A}_{f}\right) \times W_{E}$-representations. Here $\pi_{f}$ runs through irreducible admissible representations of $G\left(\mathbb{A}_{f}\right)$, the integer $a\left(\pi_{f}\right)$ is as in [24, p. 657].

Proof. Recall that

$$
\begin{aligned}
& G\left(\mathbb{Q}_{p}\right)=D^{\times} \times \mathbb{Q}_{p}^{\times}, \\
& I\left(\mathbb{Q}_{p}\right)=J_{b}\left(\mathbb{Q}_{p}\right)=G L_{n}(E) \times \mathbb{Q}_{p}^{\times}, \\
& G\left(\mathbb{A}_{f}^{p}\right)=I\left(\mathbb{A}_{f}^{p}\right) .
\end{aligned}
$$

For any irreducible representation $\Pi_{f}$ of $I\left(\mathbb{A}_{f}\right)$, let $\Pi_{p}$ (respectively $\Pi^{p}$ ) be its p-component (respectively component outside $p$ ). As in the paragraph preceding Lemma 3.1, we denote $\Pi_{p}=\Pi_{p, 0} \otimes \chi_{p}$, with $\Pi_{p, 0}$ an irreducible smooth representation of $G L_{n}(E), \chi_{p}$ a character of $\mathbb{Q}_{p}^{\times}$. Then for any irreducible representation $\rho$ of $D^{\times}$, the tensor product

$$
\rho \otimes \Pi^{p, 0}
$$

gives us an irreducible representation $\pi_{f}$ of $G\left(\mathbb{A}_{f}\right)$, and vice versa. By Lemma 3.1 we have

$$
\begin{aligned}
H_{\xi}= & \sum_{\substack{i, j \geqslant 0 \\
\Pi \subset \mathcal{A}(I)_{\xi}}}(-1)^{i+j} \underset{m}{\lim } \operatorname{Ext}_{G L_{n}(E)}^{i}\left(H_{c}^{j}\left(\mathcal{M}_{D r, m} \times \mathbb{C}_{p}, \overline{\mathbb{Q}}_{l}(n-1)\right), \Pi_{p, 0}\right) \\
& \left.\otimes r_{-\mu_{1}} \circ \varphi_{\chi_{p}}\right|_{W_{E}} \otimes \chi_{p} \otimes \Pi^{p} \\
= & \left.\sum_{\Pi \subset \mathcal{A}(I)_{\xi}} \operatorname{Mant}_{\mu_{0}}\left(\Pi_{p, 0}\right) \otimes r_{-\mu_{1}} \circ \varphi_{\chi_{p}}\right|_{W_{E}} \otimes \chi_{p} \otimes \Pi^{p} .
\end{aligned}
$$

As the notation in the proof of Lemma 3.1, we have to compute

$$
\operatorname{Mant}_{\mu_{0}}\left(\Pi_{p, 0}\right)=\sum_{i, j \geqslant 0}(-1)^{i+j} \underset{m}{\lim _{m}} \operatorname{Ext}_{G L_{n}(E)}^{i}\left(H_{c}^{j}\left(\mathcal{M}_{D r, m} \times \mathbb{C}_{p}, \overline{\mathbb{Q}}_{l}(n-1)\right), \Pi_{p, 0}\right) .
$$

First, we rewrite it as

$$
\operatorname{Mant}_{\mu_{0}}\left(\Pi_{p, 0}\right)=\sum_{\substack{i \geqslant 0 \\ k \geqslant 0}}(-1)^{i+n-1+k} \underset{m}{\underset{m}{\longrightarrow}} \operatorname{Ext}_{G L_{n}(E)}^{i}\left(H_{c}^{n-1+k}(n-1)^{\left.K_{p, 0}^{m}, \Pi_{p, 0}\right) .}\right.
$$

Apply the formula in Theorem 3.3 for $H_{c}^{n-1+k}$ (here over $\overline{\mathbb{Q}}_{l}$ we can ignore the Tate twist), then take the terms other than $\pi_{\rho}^{\leqslant k}$ out of the Ext, and then take the direct limit on $m$ we get that $\operatorname{Mant}_{\mu_{0}}\left(\Pi_{p, 0}\right)$ equals

$$
\sum_{\rho \in \operatorname{Irr}\left(D^{\times}\right)} \sum_{\substack{i \geqslant 0 \\ n_{\rho}-1 \geqslant k \geqslant 0}}(-1)^{i+n-1+k} \operatorname{Ext}_{G L_{n}(E)}^{i}\left(\pi_{\rho}^{\leqslant k}, \Pi_{p, 0}\right) \otimes \rho \otimes \sigma^{\prime}\left(J L(\rho)^{\vee}\right)|-|^{\frac{n / n_{\rho}-n}{2}-k+\frac{1-n}{2}} .
$$


Now by [8, Proposition 2.1.17], for $0 \leqslant k \leqslant n_{\rho}-1, \operatorname{Ext}_{G L_{n}(E)}^{i}\left(\pi_{\rho}^{\leqslant k}, \Pi_{p, 0}\right) \neq 0$ if and only if $\Pi_{p, 0}$ is elliptic and has the supercuspidal support $\pi_{\rho}$ and $i$ satisfies an equality which depends on $k$ and $\Pi_{p, 0}$. In this case, $\operatorname{Ext}_{G L_{n}(E)}^{i}\left(\pi_{\rho}^{\leqslant k}, \Pi_{p, 0}\right)=\overline{\mathbb{Q}}_{l}$, and since $\Pi_{p, 0}$ is preunitary for any fixed isomorphism $\overline{\mathbb{Q}}_{l} \simeq \mathbb{C}$, it has to be $\pi_{\rho}^{\leqslant 0}:=J L(\rho)$ or the local Speh representation $\pi_{\rho}^{\leqslant n_{\rho}-1}$ (see [8,5.2 p. 140]). On the other hand, we know by construction

$$
\sum_{k=0}^{n_{\rho}-1} \sigma^{\prime}\left(J L(\rho)^{\vee}\right)|-|^{\frac{n / n_{\rho}-n}{2}-k}=\sigma\left(J L(\rho)^{\vee}\right) .
$$

Therefore, we can continue the above formula as follows:

$$
\begin{aligned}
H_{\xi}= & \left.\sum_{\rho \in \operatorname{Irr}\left(D^{\times}\right)} \sum_{\substack{\Pi \subset \mathcal{A}(I) \xi \\
\Pi_{p, 0}=J L(\rho)}}(-1)^{n-1} \rho \otimes \sigma\left(J L(\rho)^{\vee}\right)|-|^{\frac{1-n}{2}} \otimes r_{-\mu_{1}} \circ \varphi_{\chi_{p}}\right|_{W_{E}} \otimes \chi_{p} \otimes \Pi^{p} \\
& +\sum_{\rho \in \operatorname{Irr}\left(D^{\times}\right)} \sum_{\substack{\Pi \subset \mathcal{A}(I)_{\xi} \\
\Pi_{p, 0}=\pi_{\rho}^{\leqslant n \rho-1}}}(-1)^{n-1+n_{\rho}-1} \rho \otimes \sigma\left(J L(\rho)^{\vee}\right)|-|^{\frac{1-n}{2}} \otimes r_{-\mu_{1}} \circ \varphi_{\chi_{p}} \mid W_{E} \otimes \chi_{p} \otimes \Pi^{p} \\
= & \sum_{\rho \in \operatorname{Irr}\left(D^{\times}\right)} \sum_{\substack{\Pi \subset \mathcal{A}(I)_{\xi} \\
\Pi_{p, 0}=J L(\rho) \text { or } \pi_{\rho}^{\leqslant n}-1}}(-1)^{d_{\rho}} \rho \otimes \sigma\left(J L(\rho)^{\vee}\right)|-|^{\frac{1-n}{2}} \otimes r_{-\mu_{1}} \circ \varphi_{\chi_{p}} \mid W_{E} \otimes \chi_{p} \otimes \Pi^{p},
\end{aligned}
$$

where $d_{\rho} \in\{0,1\}$ depends on $\rho$. For $\rho, \Pi$ occurring in the above formula, let $\pi_{f}=\rho \otimes$ $\chi_{p} \otimes \Pi^{p}$. Then $\pi_{p}=\rho \otimes \chi_{p}$, and

$$
\left.r_{-\mu} \circ \varphi_{\pi_{p}}\right|_{W_{E}}=\left.\left.r_{-\mu_{0}} \circ \varphi_{\rho}\right|_{W_{E}} \otimes r_{-\mu_{1}} \circ \varphi_{\chi_{p}}\right|_{W_{E}} .
$$

By comparing with Matsushima's formula, the above formula for $H_{\xi}$ equals

$$
\sum_{\pi_{f}} a\left(\pi_{f}\right) \pi_{f} \otimes\left(r_{-\mu} \circ \varphi_{\pi_{p}} \mid W_{E}\right)|-|^{(1-n) / 2},
$$

with the integer $a\left(\pi_{f}\right)$ as in [24, p. 657]. Note that we have

$$
a\left(\pi_{f}\right)=\sum_{\rho \in \operatorname{Irr}\left(D^{\times}\right)} \sum_{\substack{\Pi \subset \mathcal{A}(I)_{\xi} \\ \Pi_{p, 0}=J L(\rho) \text { or } \pi_{\rho}^{\leqslant n}-1 \\ \pi_{f}=\rho \otimes \chi_{p} \otimes \Pi^{p}}}(-1)^{d_{\rho}} .
$$

Here we have used the explicit description of

$$
\begin{aligned}
r_{-\mu_{0}}:{ }^{L}\left(D_{E}^{\times}\right)=G L_{n}\left(\overline{\mathbb{Q}}_{l}\right) \times( & \left.\prod_{\substack{\tau^{\prime}: E \hookrightarrow \overline{\mathbb{Q}}_{p} \\
\tau^{\prime} \neq \tau}} G L_{n}\left(\overline{\mathbb{Q}}_{l}\right)\right) \times W_{E} \longrightarrow G L_{n}\left(\overline{\mathbb{Q}}_{l}\right) \\
\left(g, g^{\prime}, \sigma\right) & \longmapsto\left(g^{-1}\right)^{t} .
\end{aligned}
$$

Hence we have

$$
\left.r_{-\mu_{0}} \circ \varphi_{\rho}\right|_{W_{E}}=\sigma(J L(\rho))^{\vee}=\sigma\left(J L(\rho)^{\vee}\right)
$$


In [27] Mantovan introduced Igusa varieties for all PEL type Shimura varieties with the related reductive groups unramified at $p$, and established a formula which describes the cohomology of each Newton strata by the cohomology of the associated Rapoport-Zink spaces and Igusa varieties. Here in our setting, the space $\mathcal{A}(I)_{\xi}$ is a substitute of the cohomology of the Igusa varieties, as one can define in a similar way to [27]. However, the space $\mathcal{A}(I)_{\xi}$ should be slightly larger, since one should have a similar formula as [42, Theorem 6.7] which says that, the cohomology of the Igusa varieties should be understood from automorphic representations of $G$ by the Jacquet-Langlands correspondence, therefore locally at $p$ the representations are only restricted in the class of all discrete series of $G L_{n}$.

\section{Deformation spaces of special $O_{D}$-modules and test functions}

We want to prove the above theorem for all $p$-adically uniformized Shimura varieties introduced in $\S 2$, i.e., $r$ can be arbitrary positive integer. To this end, we will follow an another approach: the Langlands-Kottwitz approach for these Shimura varieties at hand. We will apply Scholze's method to define some test functions by means of deformation spaces of $p$-divisible groups (cf. [37-39]). Our local setting is in the EL case as in [34], which is not included in [39] since there one restricts to the general linear groups.

We change the notations in this section. We will not make the full generality as in [34, 1.38 and Definition 3.18]. Here we will restrict ourself to the simple EL case as in [34]. The general case can be studied in the same way, or by working with each simple factor as presented here. In fact, for the purpose of this paper, only a more restricted case will be used later. Let $D$ be a central division algebra of dimension $n^{2}$ over a finite extension $F$ of $\mathbb{Q}_{p}$, with invariant $\frac{s}{n}$. Let $V$ be a finite left $D$-module. We fix a maximal order $O_{D} \subset D$ and a $O_{D}$-stable lattice $\Lambda \subset V$. These data give use the semisimple $\mathbb{Q}_{p}$-algebra $C=\operatorname{End}_{D}(V)$ with the maximal order $O_{C}=\operatorname{End}_{O_{D}}(\Lambda)$. Let $G / \mathbb{Z}_{p}$ be the algebraic group whose group of $R$-valued points is given by

$$
G(R)=\left(R \otimes_{\mathbb{Z}_{p}} O_{C}\right)^{\times}
$$

for any $\mathbb{Z}_{p}$-algebra $R$. Let $\{\mu\}$ be the conjugacy class of the cocharacter

$$
\mu: \mathbb{G}_{m} \longrightarrow G_{\overline{\mathbb{Q}}_{p}}
$$

The field of definition of $\{\mu\}$ is denoted by $E$. Fix a representative $\mu$ of $\{\mu\}$ over $\overline{\mathbb{Q}}_{p}$. We assume that only weights 0 and 1 occur in the associated decomposition of $V$ over $\overline{\mathbb{Q}}_{p}$, i.e., $V_{\overline{\mathbb{Q}}_{p}}=V_{0} \oplus V_{1}$. The isomorphism class of the subspace $V_{0}$ (and $V_{1}$ ) is defined over $E$. We make the following definition of special $O_{D}$-modules as $p$-divisible groups with suitable actions of $O_{D}$ (cf. [39, Definition 3.3] or [34, 3.23]).

Definition 4.1. Let $S$ be a scheme over $O_{E}$ on which $p$ is locally nilpotent. A special $O_{D}$-module is given by a pair $\underline{H}=(H, \iota)$ consisting of

- a $p$-divisible group $H$ over $S$;

- a homomorphism $\iota: O_{D} \rightarrow \operatorname{End}(H)$ such that 
(1) locally on $S$ there is an isomorphism of $O_{D} \otimes O_{S}$-modules between the Lie algebra of the universal vector extension of $H$ and $O_{D} \otimes_{\mathbb{Z}_{p}} O_{S}$; and

(2) the determinant condition holds true, i.e., we have an identity of polynomial functions in $a \in O_{D}$

$$
\operatorname{det}_{O_{S}}(a \mid \text { LieH })=\operatorname{det}_{E}\left(a \mid V_{0}\right) .
$$

With the above definition, many results of [39, $\S \S 3$ and 4] still hold true in our context. We just review and summarize what we need; for details we refer to [39]. Let $\underline{H}=(H, \iota)$ be a special $O_{D}$ module over a perfect field $\kappa$ of characteristic $p$, which we give a structure of an $O_{E}$-algebra via a fixed morphism $O_{E} \rightarrow \kappa$.

- The deformation functor $D e f_{\underline{H}}$ of $\underline{H}$ as a special $O_{D}$-module is pro-representable by a complete noetherian local $O_{E}$-algebra $R_{\underline{H}}$ with residue field $\kappa$.

- Let $k^{0}$ be the complete discrete valuation ring with residue field $\kappa$ that is unramified over $O_{E}$, and let $k$ be its fraction field. Then $R_{\underline{H}}$ has a structure as a $k^{0}$-algebra. Let $X_{\underline{H}}=\left(S p f R_{H}\right)^{r i g}$ be the rigid generic fiber of $S p f R_{H}$, as a rigid analytic space over $k$. Then for any open compact subgroup $K \subset G\left(\mathbb{Z}_{p}\right)$, we have a finite étale covering $X_{\underline{H}, K}$ of $X_{\underline{H}}$ parameterizing level $K$ structures.

- If $X_{\underline{H}} \neq \emptyset$, then $\kappa_{G}(b)=\mu^{\sharp}$, where $b \in B\left(G_{\mathbb{Q}_{p}}\right)$ is the $\sigma$-conjugacy class defined from the Frobenius morphism on the covariant Dieudonné module of $\underline{H}, \kappa_{G}: B\left(G_{\mathbb{Q}_{p}}\right) \longrightarrow$ $X^{*}\left(Z(\widehat{G})^{\Gamma}\right)$ is the Kottwitz map defined in $[26], \mu^{\sharp} \in X^{*}\left(Z(\widehat{G})^{\Gamma}\right)$ is the element defined from the conjugacy class of cocharacters $\mu$ as in [26]. Here $B\left(G_{\mathbb{Q}_{p}}\right)$ is the set of $\sigma$-conjugacy class in $G\left(W(\overline{\mathcal{K}})_{\mathbb{Q}}\right), \widehat{G}$ is the dual group, $Z(\widehat{G})$ its center, and $\Gamma=\operatorname{Gal}\left(\overline{\mathbb{Q}}_{p} / \mathbb{Q}_{p}\right)$.

- For any perfect field $\kappa$ of characteristic $p$ which is an $O_{E}$-algebra, there is an association by using Dieudonné module theory $\underline{H} \mapsto \delta \in G\left(W(\kappa)_{\mathbb{Q}}\right)$ which defines an injection from the set of isomorphism classes of special $O_{D}$-modules over $\kappa$ such that $X_{\underline{H}} \neq \emptyset$ into the set of $G(W(\kappa))$ - $\sigma$-conjugacy classes in $G(W(\kappa) \mathbb{Q})$ with the properties $p O_{D} \subset p \delta O_{D} \subset$ $O_{D}$ and $\kappa_{G}(p \delta)=\mu^{\sharp}$.

- We say $\underline{H}$ has controlled cohomology if $X_{\underline{H}, K}$ has controlled cohomology for all normal pro-p open subgroups $K \subset G\left(\mathbb{Z}_{p}\right)$ and all primes $l \neq p$ in the sense of [39, Definition 2.2]. Assume $\underline{H}$ has controlled cohomology. Then for any normal pro-p open subgroup $K \subset G\left(\mathbb{Z}_{p}\right)$, there is an integer $m \geqslant 1$ such that for all automorphisms $j$ of $\underline{H}$ that act trivially on $\underline{H}\left[p^{m}\right]$ the induced action on $H^{i}\left(X_{\underline{H}, K} \times \hat{\bar{k}}, \mathbb{Q}_{l}\right)$ is trivial for all $i$.

Let $I_{E} \subset W_{E}$ be the inertia subgroup of the Weil group, and fix a geometric Frobenius element Frob $\in W_{E}$. Fix some integer $j \geqslant 1$. Let $\tau \in$ Frob $^{j} I_{E} \subset W_{E}$ and $h \in C_{c}^{\infty}\left(G\left(\mathbb{Z}_{p}\right)\right)$ be a function with values in $\mathbb{Q}$. Set $t=j\left[\kappa_{E}: \mathbb{F}_{p}\right]$ where $\kappa_{E}$ is the residue field of $E$ (the standard notation for $j\left[\kappa_{E}: \mathbb{F}_{p}\right]$ should be $r$, but we have used $r$ as the copies of Drinfeld spaces in the $p$-adic uniformization). We regard $\mathbb{F}_{p^{t}}$ as the degree- $j$-extension of $\kappa_{E}$. As before, let $k$ be the unramified extension of $E$ with residue field $\mathbb{F}_{p^{t}}$. Fix the Haar measures on $G\left(\mathbb{Q}_{p}\right)$, respectively $G\left(\mathbb{Q}_{p^{t}}\right)$, that give $G\left(\mathbb{Z}_{p}\right)$ respectively $G\left(\mathbb{Z}_{p^{t}}\right)$ volume 1 . 
Definition 4.2. Let $\delta \in G\left(\mathbb{Q}_{p^{t}}\right)$. If $\delta$ is associated to some special $O_{D^{-m o d u l e}} \underline{H}$ over $\mathbb{F}_{p^{t}}$ under the above association, and if $\underline{H}$ has controlled cohomology, define

$$
\phi_{\tau, h}(\delta)=\operatorname{tr}\left(\tau \times h \mid H^{*}\left(X_{\underline{H}, K} \times \hat{\bar{k}}, \mathbb{Q}_{l}\right)\right)
$$

for any normal compact pro- $p$ open subgroup $K \subset G\left(\mathbb{Z}_{p}\right)$ such that $h$ is $K$-biinvariant. Otherwise, define $\phi_{\tau, h}(\delta)=0$. Here and in the following

$$
H^{*}\left(X_{\underline{H}, K} \times \hat{\bar{k}}, \mathbb{Q}_{l}\right)=\sum_{i \geqslant 0}(-1)^{i} H^{i}\left(X_{\underline{H}, K} \times \hat{\bar{k}}, \mathbb{Q}_{l}\right) .
$$

Proposition 4.3. (1) The function $\phi_{\tau, h}: G\left(\mathbb{Q}_{p^{t}}\right) \rightarrow \mathbb{Q}_{l}$ is well defined and takes values in $\mathbb{Q}$ independent of $l$. Its support is contained in the compact set of all $\delta \in G\left(\mathbb{Q}_{p^{t}}\right)$ satisfying $p O_{D} \subset p \delta O_{D} \subset O_{D}$ and $\kappa_{G}(p \delta)=\mu^{\sharp}$.

(2) The function $\phi_{\tau, h}$ is locally constant, so that it defines an element $\phi_{\tau, h} \in$ $C_{c}^{\infty}\left(G\left(\mathbb{Q}_{p^{t}}\right)\right)$.

Proof. Identical to the proofs of [39, Propositions 4.2 and 4.3].

We come back to the global situation as in $\S 2$. Recall we have local central division algebras $D_{i}=B_{\varpi_{i}}$ over $F_{\varpi_{i}}$ with invariant $\frac{1}{n}$ for $1 \leqslant i \leqslant r$ and the semisimple $\mathbb{Q}_{p}$-algebra $D^{\prime}$ which corresponds to the factors of $B$ at the primes $\varpi_{r+1}, \ldots, \varpi_{s}$. For each $1 \leqslant i \leqslant r$, the cocharacter $\mu_{i}$ has the form that

$$
\begin{aligned}
\mathbb{G}_{m} & \longrightarrow G_{i \overline{\mathbb{Q}}_{p}} \\
z & \mapsto\left(\left(\begin{array}{cc}
1 & \\
& z 1_{n-1}
\end{array}\right), 1_{n}, \ldots, 1_{n}\right),
\end{aligned}
$$

where $G_{i}=\operatorname{Res}_{F_{\varpi_{i}} \mid \mathbb{Q}_{p}} D_{i}^{\times}$and for any integer $d \geqslant 1,1_{d}$ is the identity $d \times d$ matrix. Recall now $E$ is the compositum of the fields $F_{\varpi_{i}}$ for $1 \leqslant i \leqslant r$. We consider the following $p$-divisible groups.

Definition 4.4. Let $S$ be a scheme over $O_{E}$ on which $p$ is locally nilpotent. A $\left(D_{1}, \ldots, D_{r}, D^{\prime}\right)$-group over $S$ is a $p$-divisible group $\underline{\widetilde{H}}=\left(\left(H_{1}, \iota_{1}\right), \ldots,\left(H_{r}, \iota_{r}\right),\left(H^{\prime}, \iota^{\prime}\right)\right)$ where

- for each $1 \leqslant i \leqslant r,\left(H_{i}, \iota_{i}\right)$ is a special $O_{D_{i}}$-module over $S$;

- $\left(H^{\prime}, \iota^{\prime}\right)$ is a $D^{\prime}$ group over $S$ in the sense of [38, Definition 4.1], i.e., an étale $p$-divisible group $H^{\prime}$ over $S$ together with an action $\iota^{\prime}: O_{D^{\prime}}^{o p} \rightarrow \operatorname{End}\left(H^{\prime}\right)$ such that $H^{\prime}[p]$ is free of rank 1 over $O_{D^{\prime}}^{o p} / p$.

Recall that there are two ways to parameterize $D^{\prime}$-groups $H^{\prime}$ over $\mathbb{F}_{p^{t}}$, the Dieudonné parametrization and the Galois parametrization. Let $\sigma_{0}$ be the absolute Frobenius of $\mathbb{Z}_{p^{t}}$. The set of isomorphism classes of such $D^{\prime}$-groups is in bijection with the set of $\left(O_{D^{\prime}} \otimes_{\mathbb{Z}_{p}} \mathbb{Z}_{p^{t}}\right)^{\times}-\sigma_{0}$ conjugacy classes in $\left(O_{D^{\prime}} \otimes_{\mathbb{Z}_{p}} \mathbb{Z}_{p^{t}}\right)^{\times}$, which is in turn bijection with the set of $O_{D^{\prime}}^{\times}$-conjugacy classes in $O_{D^{\prime}}^{\times}$by a map $\delta^{\prime} \mapsto N \delta^{\prime}$ (cf. [38, Proposition 4.2]). Let $h^{\prime} \in C_{c}^{\infty}\left(O_{D^{\prime}}^{\times}\right)$be a function which takes values in $\mathbb{Q}$ and invariant under conjugation. 
We define a function $\phi_{h^{\prime}} \in C_{c}^{\infty}\left(\left(O_{D^{\prime}} \otimes_{\mathbb{Z}_{p}} \mathbb{Z}_{p^{t}}\right)^{\times}\right)$by setting $\phi_{h^{\prime}}\left(\delta^{\prime}\right)=h^{\prime}\left(N \delta^{\prime}\right)$. Then by [38, Proposition 4.3] the functions $\phi_{h^{\prime}}$ and $h^{\prime}$ have matching (twisted) orbital integrals.

Let $\underline{\widetilde{H}}$ be a $\left(D_{1}, \ldots, D_{r}, D^{\prime}\right)$-group over $\mathbb{F}_{p^{t}}$, with $t=j\left[\kappa_{E}: \mathbb{F}_{p}\right]$. Let $G_{B}$ be the group scheme over $\mathbb{Z}_{p}$ given by

$$
G_{B}(R)=\left(O_{B} \otimes_{\mathbb{Z}_{p}} R\right)^{\times}
$$

for any $\mathbb{Z}_{p}$-algebra $R$. Then $G_{B} \times \mathbb{G}_{m}$ is a integral model of the $G_{\mathbb{Q}_{p}}$ introduced in $\S 2$. As in the single factor case, the group $\underline{\widetilde{H}}$ gives rise to an element

$$
\delta=\left(\delta_{1}, \ldots, \delta_{r}, \delta^{\prime}\right) \in G_{B}\left(\mathbb{Q}_{p^{t}}\right)=\left(D_{1} \otimes_{\mathbb{Q}_{p}} \mathbb{Q}_{p^{t}}\right)^{\times} \times \cdots \times\left(D_{r} \otimes_{\mathbb{Q}_{p}} \mathbb{Q}_{p^{t}}\right)^{\times} \times G_{D^{\prime}}\left(\mathbb{Q}_{p^{t}}\right)
$$

which is well defined up to $G_{B}\left(\mathbb{Z}_{p^{t}}\right)$ - $\sigma_{0}$-conjugacy. The deformation functor of $\underline{\tilde{H}}$ as $\left(D_{1}, \ldots, D_{r}, D^{\prime}\right)$-group is pro-representable by a $k^{0}$-algebra $R_{\widetilde{H}}$, where as before $k^{0}$ is the unramified extension of $O_{E}$ with residue field $\mathbb{F}_{p^{t}}$ and fraction field $k$. We assume

$$
X_{\underline{\widetilde{H}}}=\left(\operatorname{Spf} R_{\underline{\widetilde{\widetilde{U}}}}\right)^{r i g}
$$

non-empty, which imposes the condition $\kappa_{G_{B}}(p \delta)=\mu^{\sharp}$ on $\delta$. Moreover, for an open compact subgroup of the form $K=\left(\prod_{i=1}^{r} K_{i}\right) \times K^{\prime} \subset G_{B}\left(\mathbb{Z}_{p}\right)$, we have a product decomposition of the level $K$ covering space

$$
X_{\underline{\widetilde{H}}, K}=\left(\prod_{i=1}^{r} X_{\underline{H}_{i}, K_{i}}\right) \times G_{D^{\prime}} / K^{\prime}
$$

of $X_{\underline{\widetilde{H}}}$. Now for $\tau \in \operatorname{Frob}^{j} I_{E} \subset W_{E}, h_{1} \in C_{c}^{\infty}\left(O_{D_{1}}^{\times}\right), \ldots, h_{r} \in C_{c}^{\infty}\left(O_{D_{r}}^{\times}\right), h^{\prime} \in C_{c}^{\infty}\left(O_{D^{\prime}}^{\times}\right)$, with all these functions take values in $\mathbb{Q}$ and $h^{\prime}$ invariant under conjugation, we have defined the functions $\phi_{\tau, h_{1}} \in C_{c}^{\infty}\left(D_{1}^{\times}\left(\mathbb{Q}_{p^{t}}\right)\right), \ldots, \phi_{\tau, h_{r}} \in C_{c}^{\infty}\left(D_{r}^{\times}\left(\mathbb{Q}_{p^{t}}\right)\right)$ and $\phi_{h^{\prime}} \in$ $C_{c}^{\infty}\left(\left(O_{D^{\prime}} \otimes_{\mathbb{Z}_{p}} \mathbb{Z}_{p^{t}}\right)^{\times}\right)$. We can also define a function $\phi_{\tau, h_{1}, \ldots, h_{r}, h^{\prime}} \in C_{c}^{\infty}\left(G_{B}\left(\mathbb{Q}_{p^{t}}\right)\right)$ by

$$
\phi_{\tau, h_{1}, \ldots, h_{r}, h^{\prime}}(\delta)=\operatorname{tr}\left(\tau \times h_{1} \times \cdots \times h_{r} \times h^{\prime} \mid H^{*}\left(X_{\underline{\tilde{H}}, K} \times \hat{\bar{k}}, \mathbb{Q}_{l}\right)\right)
$$

for any normal compact pro- $p$ open subgroup $K \subset G_{B}\left(\mathbb{Z}_{p}\right)$ such that $\left(h_{1}, \ldots, h_{r}, h^{\prime}\right)$ is $K$-biinvariant, if $\delta$ is associated to a controlled $\left(D_{1}, \ldots, D_{r}, D^{\prime}\right)$-group $\underline{\widetilde{H}}$, otherwise define $\phi_{\tau, h_{1}, \ldots, h_{r}, h^{\prime}}(\delta)=0$. Then it is easy to see that for $\delta=\left(\delta_{1}, \ldots, \delta_{r}, \delta^{\prime}\right)$ we have

$$
\phi_{\tau, h_{1}, \ldots, h_{r}, h^{\prime}}(\delta)=\phi_{\tau, h_{1}}\left(\delta_{1}\right) \cdots \phi_{\tau, h_{r}}\left(\delta_{r}\right) \phi_{h^{\prime}}\left(\delta^{\prime}\right) .
$$

Our global PEL situation actually puts us in the quasi-EL case in the following sense, which is the analogue of the quasi-EL case of $[39, \S 3]$ in our context. Let $S$ be a locally noetherian scheme on which $p$ is locally nilpotent. Recall for an abelian scheme $A$ which comes from an $S$-valued point of the moduli space $S_{K^{p}}$ defined in $\S 2$, the associated $p$-divisible group has the decomposition

$$
A\left[p^{\infty}\right]=\underline{\tilde{H}} \oplus \underline{\tilde{H}}^{D},
$$

with $\underline{\widetilde{H}}$ a $\left(D_{1}, \ldots, D_{r}, D^{\prime}\right)$-group, $\underline{\widetilde{H}}^{D}$ its dual. Then one can also formulate a definition of $p$-divisible groups over $S$ with the same additional (PEL) structures as those in the form $A\left[p^{\infty}\right]$ for $A$ coming from an $S$-point of $S_{K}$ (cf. [39, Definition 3.3]). We may call 
these additional structures as quasi-EL additional structures. Such a $p$-divisible group with quasi-EL additional structures is given by a quadruple $\underline{H}=(H, \iota, \lambda, \mathbb{L})$ over $S$, with $\lambda: H \stackrel{\sim}{\longrightarrow} H^{D} \otimes \mathbb{L}$ a (twisted) principal polarization and $\mathbb{L}$ is a one-dimensional smooth $\mathbb{Z}_{p}$-local system. It is equivalent to giving a $\left(D_{1}, \ldots, D_{r}, D^{\prime}\right)$-group $\underline{\widetilde{H}}_{0}$ and an one-dimensional smooth $\mathbb{Z}_{p}$-local system $\mathbb{L}$ over $S$. The relation between the two is given by the equality $H=H_{0} \oplus H_{0}^{D} \otimes \mathbb{L}$. In particular, for such a $p$-divisible group $\underline{H}$ over $\mathbb{F}_{p^{t}}$, we get an element

$$
\delta=\left(\delta_{0}, p^{-1} d\right) \in G\left(\mathbb{Q}_{p^{t}}\right)=G_{B}\left(\mathbb{Q}_{p^{t}}\right) \times \mathbb{Q}_{p^{t}}^{\times}
$$

where $\mathbb{L}$ corresponds to $d$ as in $[39, \S 3]$. Then for any open compact subgroup of the form

$$
K=K_{0} \times\left(1+p^{m} \mathbb{Z}_{p}\right) \subset G\left(\mathbb{Z}_{p}\right)=G_{B}\left(\mathbb{Z}_{p}\right) \times \mathbb{Z}_{p}^{\times}
$$

with $m \geqslant 1$, we have a product decomposition

$$
X_{\underline{H}, K}=X_{\underline{\tilde{H}}_{0}, K_{0}} \times X_{\mathbb{L}, m},
$$

where $X_{\mathbb{L}, m}$ parameterizes isomorphisms between $\mathbb{L} \otimes \mu_{p^{m}}$ and $\mathbb{Z} / p^{m} \mathbb{Z}$. Similarly for $\tau \in$ Frob $^{j} I_{E} \subset W_{E},\left(h_{0}, h_{\mathbb{G}_{m}}\right) \in C_{c}^{\infty}\left(G_{B}\left(\mathbb{Z}_{p}\right)\right) \times C_{c}^{\infty}\left(\mathbb{Z}_{p}^{\times}\right)$with values in $\mathbb{Q}$, we can define a test

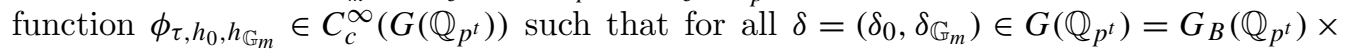
$\mathbb{Q}_{p^{t}}^{\times}$, we have

$$
\phi_{\tau, h_{0}, h_{\mathbb{G} m}}(\delta)=\phi_{\tau, h_{0}}\left(\delta_{0}\right) \phi_{\tau, h_{\mathbb{G}_{m}}}\left(\delta_{\mathbb{G}_{m}}\right)
$$

where $\phi_{\tau, h_{\mathbb{G} m}}$ is the function with support on $p^{-1} \mathbb{Z}_{p^{t}}^{\times}$defined by

$$
\phi_{\tau, h_{\mathbb{G}_{m}}}\left(\delta_{\mathbb{G}_{m}}\right)=h\left(\operatorname{Art}_{\mathbb{Q}_{p}}(\tau) N \delta_{\mathbb{G}_{m}}\right)
$$

where $\operatorname{Art}_{\mathbb{Q}_{p}}: W_{\mathbb{Q}_{p}} \rightarrow \mathbb{Q}_{p}^{\times}$is the local reciprocity map sending a geometric Frobenius element to a uniformizer. As in [39, Remark 4.11], for all characters $\chi: \mathbb{Q}_{p}^{\times} \rightarrow \mathbb{C}^{\times}$, we have the identity

$$
\operatorname{tr}\left(\phi_{\tau, h_{\mathbb{G} m}} \mid \chi \circ N_{\mathbb{Q}_{p} t \mathbb{Q}_{p}}\right)=\operatorname{tr}\left(\tau^{-1} \mid \chi \circ \operatorname{Art} \mathbb{Q}_{p}\right) \operatorname{tr}(h \mid \chi),
$$

where $N_{\mathbb{Q}_{p} \mid \mathbb{Q}_{p}}: \mathbb{Q}_{p^{t}}^{\times} \rightarrow \mathbb{Q}_{p}^{\times}$is the norm map.

\section{Counting points modulo $p$}

We return to the situation of $\S 2$. We are going to adapt Kottwitz's method to count points in $S_{K^{p}}\left(\mathbb{F}_{p^{t}}\right)$ and compute the cohomology of Shimura varieties. Before going into the details, we say more about the moduli spaces $S_{K}{ }^{p}$.

We know that

$$
S_{K^{p}} \times O_{E} E \simeq \coprod_{k e r^{1}(\mathbb{Q}, G)} S h_{K_{p}^{0} K^{p}}
$$

where $K_{p}^{0} \subset G\left(\mathbb{Q}_{p}\right)$ is the maximal open compact subgroup. Now for any open compact subgroup $K_{p} \subset G\left(\mathbb{Z}_{p}\right)$, we have a finite étale cover $\pi_{K_{p} K^{p}}: S_{K_{p} K^{p}} \rightarrow S_{K^{p}} \times O_{E} E$ which parameterizes $K_{p}$-orbits of isomorphisms between $O_{B} \otimes \mathbb{Z}_{p}$ and the $p$-adic Tate module $T_{p} A$ of $A$, compatible with the additional structures. In such a way we get a tower of 
varieties equipped with an action of $G\left(\mathbb{Z}_{p}\right) \times G\left(\mathbb{A}_{f}^{p}\right)$ by Hecke correspondences. Moreover, we have isomorphisms

$$
S_{K_{p} K^{p}} \simeq \coprod_{k e r^{1}(\mathbb{Q}, G)} S h_{K_{p} K^{p}}
$$

compatible with the Hecke correspondences and the maps to $S_{K^{p}} \times{ }_{O_{E}} E$ (cf. [39, Proposition 5.3]).

Fix a prime $l \neq p$. Let $\xi$ be a finite-dimensional algebraic representation of $G$ over $\overline{\mathbb{Q}}_{l}$. Then as usual we get local systems $\mathcal{L}_{\xi}$ on $S_{K_{p} K^{p}}$ to which the action of the Hecke correspondences extend. By abuse of notation we will not put level subscripts on them. We can define the $\ell$-adic cohomology of the tower $S_{K_{p} K^{p}}$ with coefficients in the local systems $\mathcal{L}_{\xi}$ by

$$
\widetilde{H}_{\xi}=\sum_{i \geqslant 0}(-1)^{i} \underset{K_{p}, K^{p}}{\lim _{\longrightarrow}} H^{i}\left(S_{K_{p} K^{p}} \times \overline{\mathbb{Q}}_{p}, \mathcal{L}_{\xi}\right) .
$$

This is a virtual representation of $G\left(\mathbb{Z}_{p}\right) \times G\left(\mathbb{A}_{f}^{p}\right) \times W_{E}$. We have the equality

$$
\widetilde{H}_{\xi}=\bigoplus_{k e r^{1}(\mathbb{Q}, G)} H_{\xi}
$$

which is compatible with the actions of $W_{E}$ and $G\left(\mathbb{Z}_{p}\right) \times G\left(\mathbb{A}_{f}^{p}\right)$, where $H_{\xi}$ was defined in $\S 3$.

Now let $x \in S_{K^{p}}\left(\mathbb{F}_{p^{t}}\right)$, write $\underline{H}$ as the associated $p$-divisible group with quasi-EL additional structures over $\mathbb{F}_{p^{t}}$ defined in $\S 4$. Let $k$ be the complete unramified extension of $E$ with residue field $\mathbb{F}_{p^{t}}$. By Serre-Tate theorem, we know that there is an isomorphism of complete local rings $\widehat{O}_{S_{K}, x} \simeq R_{\underline{H}}$, where $R_{\underline{H}}$ is the deformation ring of $\underline{H}$. In particular, we have the natural embedding $X_{\underline{H}} \hookrightarrow S_{K_{p}^{0} K^{p}}^{\text {rig }} \times k$. Moreover, for any open compact subgroup $K_{p} \subset G\left(\mathbb{Z}_{p}\right)$ we have the pullback diagram

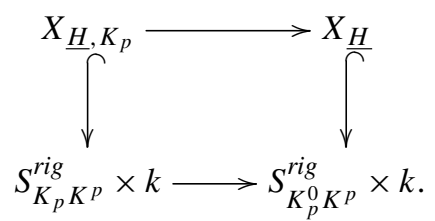

Here for any $K_{p} \subset G\left(\mathbb{Z}_{p}\right), S_{K_{p} K^{p}}^{\text {rig }}$ is the rigid analytic space over $E$ associated to $S_{K_{p} K^{p}}$. In particular, $\underline{H}$ has controlled cohomology and for all $i \in \mathbb{Z}$ we have a $\operatorname{Gal}(\bar{k} / k)$-equivariant isomorphism

$$
\left(R^{i} \psi \pi_{K_{p} K^{p_{*}}} \mathbb{Q}_{l}\right)_{\bar{x}} \simeq H^{i}\left(X_{\underline{H}, K_{p}} \times \hat{\bar{k}}, \mathbb{Q}_{l}\right),
$$

where $\pi_{K_{p} K^{p}}: S_{K_{p} K^{p}} \rightarrow S_{K_{p}^{0} K^{p}}$ is the natural projection, and $\psi$ is the nearby cycle functor for the scheme $S_{K}$.

The $p$-adic uniformization gives us the description of the set of $\overline{\mathbb{F}}_{p}$-points of $S_{K^{p}}$ :

$$
S_{K^{p}}\left(\overline{\mathbb{F}}_{p}\right)=\coprod_{\operatorname{ker}^{1}(\mathbb{Q}, G)} I(\mathbb{Q}) \backslash X_{p} \times X^{p},
$$


where $X_{p}=\mathcal{M}\left(\overline{\mathbb{F}}_{p}\right)$ with $\mathcal{M}$ the pro-formal scheme over $O_{E}$ associated to the formal Rapoport-Zink space $\widehat{\mathcal{M}}$ as in [34, Definition 3.51], $X^{p}=G\left(\mathbb{A}_{f}^{p}\right) / K^{p}$, and the Frobenius action on the left hand side induces the natural Frobenius action on $X_{p}$. The set $X_{p}$ can be described as a set of some suitable Dieudonné lattices, and after fixing a choice of a lattice we can identify $X_{p}$ with a subset of $G(L) / G\left(O_{L}\right)$ where $L=W\left(\overline{\mathbb{F}}_{p}\right)_{\mathbb{Q}}$. As $K^{p}$ varies, this bijection is compatible with the action of $G\left(\mathbb{A}_{f}^{p}\right)$. Note for our group $G$, if $K^{p}$ sufficiently small we have $Z(\mathbb{Q}) \cap K=1$, where $Z \subset G$ is the center and $K=K_{p}^{0} K^{p}$. From now on, we take a sufficiently small $K^{p}$ such that $Z(\mathbb{Q}) \cap K=1$, and such that of $[25,1.3 .7$ and 1.3.8] hold (the Proof of Lemma 5.5 in [29] works for the group $I$, as one sees easily). Passing to the finite level, by the method of [25] we have

$$
S_{K^{p}}\left(\mathbb{F} p^{t}\right)=\coprod_{k e r^{1}(\mathbb{Q}, G)} \coprod_{\varepsilon} I_{\varepsilon}(\mathbb{Q}) \backslash A_{\varepsilon},
$$

where $\varepsilon$ runs through the set of conjugacy classes in $I(\mathbb{Q}), I_{\varepsilon}$ is the centralizer of $\varepsilon$ in $I$, and $A_{\varepsilon}$ is defined as follows:

$$
\begin{aligned}
A_{\varepsilon} & =\operatorname{Fix}\left(F r^{j} \varepsilon^{-1} \mid X_{p}\right) \times \operatorname{Fix}\left(\varepsilon \mid X^{p}\right) \\
& =: X_{p}(\varepsilon) \times X^{p}(\varepsilon) .
\end{aligned}
$$

Here $F r$ is the Frobenius automorphism on $X_{p}$ over $E$. The set $A_{\varepsilon}$ could be empty. However, when it is non-empty, the set $\bigsqcup_{k e r}{ }_{(\mathbb{Q}, G)} I_{\varepsilon}(\mathbb{Q}) \backslash A_{\varepsilon}$ has a moduli explanation as follows. Let $x_{0}=\left(A_{x_{0}}, \iota_{x_{0}}, \lambda_{x_{0}}, \bar{\eta}_{x_{0}}^{p}\right) \in S_{K}\left(\mathbb{F}_{p^{t}}\right)$ be any point with associated $\varepsilon$. Then the set of points in $S_{K}^{p}\left(\mathbb{F}_{p^{t}}\right)$ for which the associated abelian varieties are isogenious to $\left(A_{x_{0}}, \iota_{x_{0}}, \lambda_{x_{0}}\right)$ over $\mathbb{F}_{p^{t}}$ is in bijection with $\bigsqcup_{k e r^{1}(\mathbb{Q}, G)} I_{\mathcal{E}}(\mathbb{Q}) \backslash A_{\varepsilon}$. As in the case of $X_{p}$, after fixing some choice of some lattice, the set $X_{p}(\varepsilon)$ can be identified with a subset of $G\left(\mathbb{Q}_{p^{t}}\right) / G\left(\mathbb{Z}_{p^{t}}\right)$.

For $\varepsilon \in I(\mathbb{Q})$ such that $A_{\varepsilon}$ is non-empty, we can associate to it a conjugacy class $\gamma \in G\left(\mathbb{A}_{f}^{p}\right)$ that is stably conjugate to $\varepsilon$ by the embedding $I(\mathbb{Q}) \subset I\left(\mathbb{A}_{f}^{p}\right)=G\left(\mathbb{A}_{f}^{p}\right)$, and a $\sigma$-conjugacy class $\delta \in G\left(\mathbb{Q}_{p^{t}}\right)$ such that $N \delta$ is stably conjugate to $\varepsilon$ (cf. [32, Theorem 4.11] and $[31$, p. 689]). The characterization of the $\sigma$-conjugacy class $\delta$ is as follows. Assume that $A_{\varepsilon}$ is non-empty, then there is a $\delta \in G\left(\mathbb{Q}_{p^{t}}\right)$ such that there exists $c \in G(L)$ with $\delta=c b \sigma(c)^{-1}$ and $N \delta=c \varepsilon c^{-1}$; here $N \delta$ is as in Definition 5.2 and $b \times \sigma$ is the $\sigma$-linear map $V^{-1}$ on the covariant rational Dieudonné module. The $\sigma$-conjugacy class of such a $\delta \in G\left(\mathbb{Q}_{p^{t}}\right)$ is uniquely determined by the $I(\mathbb{Q})$-conjugacy class of $\varepsilon$. If $A_{\varepsilon}$ is non-empty, we can also get $(\gamma, \delta)$ by geometric means. A point $x \in S_{K}\left(\mathbb{F}_{p^{t}}\right)$ with associated $\varepsilon$ gives rise to a $c$-polarized virtual abelian variety $\left(A_{x}, \iota_{x}, \lambda_{x}\right)$ over $\mathbb{F}_{p^{t}}$ (cf. [39, Definition 6.1] or $[25, \S 10])$. By the existence of a level structure of type $K^{p}$, we know that for all $l \neq p$, the rational $l$-adic Tate module $V_{l}\left(A_{x}\right)$ is isomorphic to $V \otimes \mathbb{Q}_{l}$; fixing an isomorphism, the Frobenius morphism $\pi_{A_{x}}$ gives rise to a $B$-linear automorphism of $V_{l}$; we define $\gamma_{l} \in G\left(\mathbb{Q}_{l}\right)$ as its inverse. Its conjugacy class is well defined, and these elements define a conjugacy class $\gamma \in G\left(\mathbb{A}_{f}^{p}\right)$. The associated $p$-divisible group $H=A_{x}\left[p^{\infty}\right]$ then gives an element $\delta \in G\left(\mathbb{Q}_{p^{t}}\right)$ well defined up to $\sigma$-conjugation by $G\left(\mathbb{Z}_{p^{t}}\right)$. It satisfies the equality $\kappa_{G}(p \delta)=\mu^{\sharp}$.

Now we get the first form the trace formula. Fix the Haar measures on $G\left(\mathbb{Q}_{p}\right)$, respectively $G\left(\mathbb{Q}_{p^{t}}\right)$, that give $G\left(\mathbb{Z}_{p}\right)$, respectively $G\left(\mathbb{Z}_{p^{t}}\right)$, volume 1 . 
Proposition 5.1. With the notations above, we have the following equality:

$$
\operatorname{tr}\left(\tau \times h f^{p} \mid H_{\xi}\right)=\sum_{\varepsilon} \operatorname{vol}\left(I_{\varepsilon}(\mathbb{Q}) \backslash I_{\varepsilon}\left(\mathbb{A}_{f}\right)\right) O_{\gamma}\left(f^{p}\right) T O_{\delta \sigma}\left(\phi_{\tau, h}\right) \operatorname{tr} \xi(\varepsilon),
$$

where $\varepsilon$ runs over the set of conjugacy classes of $I(\mathbb{Q})$ such that $A_{\varepsilon}$ is non-empty.

Proof. This formula comes from the application of Lefschetz trace formula [45] and the description of the set of $\mathbb{F}_{p^{t}}$-points. The local term of each fixed point in the isogeny class associated to $\varepsilon$ is $\phi_{\tau, h}(\delta) \operatorname{tr} \xi(\varepsilon)$. The contribution of fixed points in this isogeny class is given by

$$
\operatorname{vol}\left(I_{\varepsilon}(\mathbb{Q}) \backslash I_{\varepsilon}\left(\mathbb{A}_{f}\right)\right) O_{\gamma}\left(f^{p}\right) T O_{\delta \sigma}\left(\phi_{\tau, h}\right) \operatorname{tr} \xi(\varepsilon) .
$$

For a more detailed proof, see [41, Proposition 5.1].

We want to construct a 'Kottwitz triple' attached to the above $\varepsilon$. Recall the usual definition of a Kottwitz triple for our reductive group $G$ over $\mathbb{Q}$.

Definition 5.2. Let $j \geqslant 1$. Set $t=j\left[\kappa_{E}: \mathbb{F}_{p}\right]$ with $\kappa_{E}$ as the residue field of $E$. A degree- $j$-Kottwitz triple $\left(\gamma_{0} ; \gamma, \delta\right)$ consists of

- a semisimple stable conjugacy class $\gamma_{0} \in G(\mathbb{Q})$;

- a conjugacy class $\gamma \in G\left(\mathbb{A}_{f}^{p}\right)$ that is stably conjugate to $\gamma_{0}$;

- a $\sigma$-conjugacy class $\delta \in G\left(\mathbb{Q}_{p^{t}}\right)$ such that $N \delta:=\delta \sigma(\delta) \cdots \sigma^{t-1}(\delta)$ is stably conjugate to $\gamma_{0}$;

satisfying

(1) $\gamma_{0}$ is elliptic in $G(\mathbb{R})$;

(2) $\kappa_{G_{\mathbb{Q} p}}(p \delta)=\mu^{\sharp}$ in $X^{*}\left(Z(\widehat{G})^{\Gamma}\right)$, where $\Gamma$ is the absolute Galois group of $\mathbb{Q}_{p}$.

The conjugacy class of $N \delta$ is stable under the Galois group $\Gamma$. However, since $G_{\mathbb{Q}_{p}}$ is not quasi-split, it can happen that this conjugacy class contains no element of $G\left(\mathbb{Q}_{p}\right)$ (cf. [21]). This is an obstruction to obtain a $\gamma_{0} \in G(\mathbb{Q})$ such that $\left(\gamma_{0} ; \gamma, \delta\right)$ forms a Kottwitz triple for $G$. In fact, it is the only obstruction, since we have the following lemma.

Lemma 5.3. If the conjugacy class of $N \delta$ contains an element of $G\left(\mathbb{Q}_{p}\right)$, then we can find an element $\gamma_{0} \in G(\mathbb{Q})$ such that $\left(\gamma_{0} ; \gamma, \delta\right)$ forms a Kottwitz triple for $G$.

Proof. Let $(A, \iota, \lambda)$ be a $c$-polarized virtual abelian variety over $\mathbb{F}_{p^{t}}$ coming from a

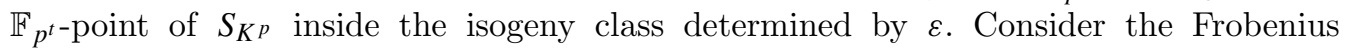
morphism $\pi_{A} \in \operatorname{End}_{B}(A)$. Then $F\left(\pi_{A}\right)$ is a CM field, which is also the center of the division algebra $\operatorname{End}_{B}(A)$. The Rosati involution $*$ on $\operatorname{End}_{B}(A)$ induced by $\lambda$ preserves $F\left(\pi_{A}\right)$. Since by assumption, the conjugacy class of $N \delta$ is stable under the Galois group $\Gamma$, we have an embedding $F\left(\pi_{A}\right) \subset B^{o p p}$. Moreover, by the definition of the moduli problem, the two involutions $*$ and $\sharp$ are compatible under this embedding. Then we have $\pi_{A} \in G(\mathbb{Q})$ under the above embedding. We can take $\gamma_{0}=\pi_{A}^{-1}$. The conditions in Definition 5.2 for $\left(\gamma_{0} ; \gamma, \delta\right)$ being a Kottwitz triple can be verified as in $[25, \S 14]$.

The converse of this lemma is clearly true. In particular, for our group $G$ which is not quasi-split at $p$, the set of Kottwitz triples is not enough for parameterizing all 
the $\mathbb{F}_{p^{t}}$-points of our Shimura varieties. Maybe it is possible to define some generalized Kottwitz triples by introducing some suitable inner form of $G$, such that these generalized Kottwitz triples parameterize all the points on the Shimura varieties over finite fields. But we will not pursue this subject here. Now the key point is the following theorem, which can be viewed as a generalization of [32, Conjecture 5.7] which was proved in the maximal level case by Waldspurger. See also [33, Conjecture 10.2] in the more general setting.

Theorem 5.4. If the conjugacy class of $N \delta$ does not contain an element of $G\left(\mathbb{Q}_{p}\right)$, then for any $j \geqslant 1, \tau \in$ Frob $^{j} I_{E}$ and any $h \in C_{c}^{\infty}\left(G\left(\mathbb{Z}_{p}\right)\right)$, we have the twisted orbital integral of the test function $\phi_{\tau, h}$ vanishes

$$
T O_{\delta \sigma}\left(\phi_{\tau, h}\right)=0
$$

In particular, there is a function $f_{\tau, h} \in C_{c}^{\infty}\left(G\left(\mathbb{Q}_{p}\right)\right)$ which has matching (twisted) orbital integrals with $\phi_{\tau, h} \in C_{c}^{\infty}\left(G\left(\mathbb{Q}_{p^{t}}\right)\right)$.

Proof. By the properties of the test function $\phi_{\tau, h}$, we can assume we are in the local quasi-EL case such that $G\left(\mathbb{Q}_{p}\right)=D^{\times} \times \mathbb{Q}_{p}^{\times}$, where $D$ is a central division algebra with invariant $\frac{1}{n}$ over a finite extension $E$ of $\mathbb{Q}_{p}$. Then the test functions $\phi_{\tau, h}$ are defined for the Shimura varieties which were studied in $\S 2$ at $p$. With the notation of $\S 2$, we have specially simple form: $r=1$ and $G_{D^{\prime}}=1$. By Proposition 5.1, we have the formula

$$
\operatorname{tr}\left(\tau \times h f^{p} \mid H_{\xi}\right)=\sum_{\varepsilon, A_{\varepsilon} \neq \emptyset} \operatorname{vol}\left(I_{\varepsilon}(\mathbb{Q}) \backslash I_{\varepsilon}\left(\mathbb{A}_{f}\right)\right) O_{\gamma}\left(f^{p}\right) T O_{\delta \sigma}\left(\phi_{\tau, h}\right) \operatorname{tr} \xi(\varepsilon),
$$

where $\varepsilon$ runs over the set of conjugacy classes of $I(\mathbb{Q})$ such that $A_{\varepsilon} \neq \varnothing$, with the associated (twisted) conjugacy classes $\gamma$ and $\delta$ as above. Recall that in the above situation $I_{\mathbb{Q}_{p}}=\left(\operatorname{Res}_{E \mid \mathbb{Q}_{p}} G L_{n}\right) \times \mathbb{G}_{m}$. Let $G^{*}=I_{\mathbb{Q}_{p}}$ be the quasi-split inner form of $G$ over $\mathbb{Q}_{p}$. Then we have the norm map

$$
\begin{aligned}
N & :\left\{\sigma-\text { conjugacy classes in } G\left(\mathbb{Q}_{p^{t}}\right)\right\} \\
& \longrightarrow\left\{\text { conjugacy classes in } G^{*}\left(\mathbb{Q}_{p}\right)=G L_{n}(E) \times \mathbb{Q}_{p}^{\times}\right\}
\end{aligned}
$$

from which we can define a transfer $f_{\tau, h}^{*} \in C_{c}^{\infty}\left(G^{*}\left(\mathbb{Q}_{p}\right)\right)$ cf. [1, 32], such that for any conjugacy class $\left\{\gamma_{p}\right\}$ not in the image of $N$ we have

$$
O_{\gamma_{p}}\left(f_{\tau, h}^{*}\right)=0
$$

and for $\left\{\gamma_{p}\right\}=N\{\delta\}$ we have

$$
O_{\gamma_{p}}\left(f_{\tau, h}^{*}\right)=e(\delta) T O_{\delta \sigma}\left(\phi_{\tau, h}\right)
$$

where $e(\delta)$ is the Kottwitz sign of $G_{\delta \sigma}$. By construction, for $\delta$ coming from $\varepsilon$, we have $N\{\delta\}=\left\{\gamma_{p}\right\}$ where $\left\{\gamma_{p}\right\}$ is the image of $\varepsilon$ in $I\left(\mathbb{Q}_{p}\right)$. Then we can rewrite the above formula as

$$
\operatorname{tr}\left(\tau \times h f^{p} \mid H_{\xi}\right)=\sum_{\varepsilon} \operatorname{vol}\left(I_{\varepsilon}(\mathbb{Q}) \backslash I_{\varepsilon}\left(\mathbb{A}_{f}\right)\right) O_{\gamma}\left(f^{p}\right) e(\delta) O_{\gamma_{p}}\left(f_{\tau, h}^{*}\right) \operatorname{tr} \xi(\varepsilon) .
$$


Note in particular by definition of $f_{\tau, h}^{*}$, in the above sum $\varepsilon$ runs over the set of all conjugacy classes of $I(\mathbb{Q})$. We identify the center $Z \subset G$ as a subgroup of $I$ and $I_{\varepsilon}$. Recall the Tamagawa number of $I_{\varepsilon}$ is defined by

$$
\tau\left(I_{\varepsilon}\right)=\operatorname{vol}\left(I_{\varepsilon}(\mathbb{Q}) A_{G}(\mathbb{R})^{0} \backslash I_{\varepsilon}(\mathbb{A})\right),
$$

where $A_{G}$ is the split component of $Z$ and $A_{G}(\mathbb{R})^{0}$ is the connected component containing the identity element. So the factor $\operatorname{vol}\left(I_{\varepsilon}(\mathbb{Q}) \backslash I_{\varepsilon}\left(\mathbb{A}_{f}\right)\right)$ equals

$$
\tau\left(I_{\varepsilon}\right) \operatorname{vol}\left(A_{G}(\mathbb{R})^{0} \backslash I_{\varepsilon}(\mathbb{R})\right)^{-1} .
$$

Let $f_{\infty}$ be a pseudo-coefficient on $I(\mathbb{R})$ for $\check{\xi}$. Denote the image of $\varepsilon$ in $I(\mathbb{R})$ by $\gamma_{\infty}$. Then as $[24$, p. 659] (recall that the group $I(\mathbb{R})$ is compact modulo center) we have

$$
O_{\gamma_{\infty}}\left(f_{\infty}\right)=e\left(\gamma_{\infty}\right) \operatorname{vol}\left(A_{G}(\mathbb{R})^{0} \backslash I_{\varepsilon}(\mathbb{R})\right)^{-1} \operatorname{tr} \xi(\varepsilon),
$$

where $e\left(\gamma_{\infty}\right)$ is the Kottwitz sign of $\boldsymbol{I}_{\mathbb{R}}$. As in [31, p. 690] or [35, $\left.\S 10\right]$, we have $e(\delta)=$ $e\left(\gamma_{\infty}\right)$. Therefore, we get

$$
\operatorname{tr}\left(\tau \times h f^{p} \mid H_{\xi}\right)=\sum_{\varepsilon} \tau\left(I_{\varepsilon}\right) O_{\gamma}\left(f^{p}\right) O_{\gamma_{p}}\left(f_{\tau, h}^{*}\right) O_{\gamma_{\infty}}\left(f_{\infty}\right)
$$

Now we apply the simple trace formula for the group $I$ and the function $f=f^{p} f_{\tau, h}^{*} f_{\infty}$. The above equals

$$
\sum_{\pi} m(\pi) \operatorname{tr} \pi(f)
$$

where $\pi$ runs over the automorphic representations of $I(\mathbb{A})$ whose central character is the inverse of that of $\xi$ on $A_{G}(\mathbb{R})^{0}$.

On the other hand, we have known by Theorem 3.4

$$
H_{\xi}=\sum_{\pi_{f}} a\left(\pi_{f}\right) \pi_{f} \otimes\left(r_{-\mu} \circ \varphi_{\pi_{p}} \mid W_{E}\right)|-|^{(1-n) / 2}
$$

as virtual $G\left(\mathbb{A}_{f}\right) \times W_{E}$-representations. Now by the comparison of this formula with the above trace formula, we can conclude that for any irreducible smooth representation $\pi_{p}=\pi_{p}^{0} \otimes \chi_{p}$ of $G^{*}\left(\mathbb{Q}_{p}\right)=G L_{n}(E) \times \mathbb{Q}_{p}^{\times}$, if $\pi_{p}^{0}$ is not a discrete series, i.e., it does not come from an irreducible smooth representation of $D^{\times}$, then

$$
\operatorname{tr} \pi_{p}\left(f_{\tau, h}^{*}\right)=0 .
$$

Indeed, for any such $\pi_{p}$, suppose first that we can find an automorphic representation $\pi$ of $I$ with the $p$-component as $\pi_{p}$ and $m(\pi) \neq 0$. Take compact open subgroups $K^{p} \subset$ $I\left(\mathbb{A}_{f}^{p}\right)=G\left(\mathbb{A}_{f}^{p}\right), K_{p} \subset I\left(\mathbb{Q}_{p}\right)$ such that $\pi_{f}^{p}$ has $K^{p}$-invariants and $\pi_{p}$ has $K_{p}$-invariants. Let $K=K^{p} K_{p}$. Then there are only finitely finitely irreducible admissible representations $\pi_{f}^{\prime}$ of $I\left(\mathbb{A}_{f}\right)$ such that $\pi_{f}^{\prime}$ occurs as the finite adelic component of an automorphic representation with central character the inverse of $\xi$ on $A_{G}(\mathbb{R})^{0}, \pi_{f}^{\prime}$ has $K$-invariants, and $m\left(\pi^{\prime}\right) \neq 0$. One can then find a function $f^{p} \in C_{c}^{\infty}\left(I\left(\mathbb{A}_{f}^{p}\right)\right)$ biinvariant under $K^{p}$ with $\operatorname{tr}\left(f^{p} \mid \pi_{f}^{p}\right)=1$ and such that whenever $\pi_{f}^{\prime}$ is an irreducible admissible representation of $I\left(\mathbb{A}_{f}\right)$ with $\operatorname{tr}\left(\pi_{f}^{\prime}\right)^{p}\left(f^{p}\right) \neq 0$, with $\left(\pi_{f}^{\prime}\right)^{p}$ has invariants under $K^{p}$, then $\left(\pi_{f}^{\prime}\right)^{p} \simeq \pi_{f}^{p}$ 
(which implies $\pi_{f}^{\prime}=\pi_{f}$ by [15, Lemma 3]. In fact the situation of [15] is under more restrictive hypotheses, but the same proof applies). Take such an $f^{p}$. Recall that by our choice of $f_{\infty}$ we have $\operatorname{tr} \pi_{\infty}\left(f_{\infty}\right)=1$. Then the right hand of the trace formula has only one term

$$
m(\pi) \operatorname{tr} \pi_{p}\left(f_{\tau, h}^{*}\right)
$$

which has to be zero according to the description of $H_{\xi}$.

For the general case, we use the facts that the set of local $p$-components of automorphic representations of $I$ is Zariski dense in the Bernstein variety of $G^{*}\left(\mathbb{Q}_{p}\right)$; see for example [42, Proposition 3.1]. More precisely, let

$$
\mathfrak{z}_{2}=\coprod_{(L, D) \in \mathfrak{S}\left(G L_{n}(E)\right)} V(L, D)
$$

be the Bernstein variety of $G L_{n}(E)$ as introduced in $[9$, A.4]. Here we use the notation of 2.1 of [42]. This is the discrete series variant of the usual Bernstein variety $\mathfrak{z}$. Let $\operatorname{Irr}\left(G L_{n}(E)\right)$ be the set of isomorphism classes of irreducible smooth representations of $G L_{n}(E)$. Then thanks to the Bernstein-Zelevinsky classification for $G L_{n}(E)$, we get a map with finite fibers

$$
r: \operatorname{Irr}\left(G L_{n}(E)\right) \rightarrow \mathfrak{z}_{2},
$$

such that its composition with the finite map $\mathfrak{z}_{2} \rightarrow \mathfrak{z}$ (cf. [42, Remark 2.2], and see also the example of A.4.e in p. 64 of [9] for an explicit description of this finite map) is the usual map $\operatorname{Irr}\left(G L_{n}(E)\right) \rightarrow \mathfrak{z}$ sending a representation to its supercuspidal support. By the classification of discrete series in [46], the set of isomorphism classes of discrete series of $G L_{n}(E)$ is exactly the inverse image under $r$ of the components $V(L, D)$ with $L=G$ (see also [42, Example 2.3]). Take any $(L, D) \in \mathfrak{S}\left(G L_{n}(E)\right)$ with $L \neq G$. Proposition 3.1 of [42] says that the set of local $p$-components of automorphic representations of $I$ (which are non-discrete series)

$$
Y=\left\{x \in V(L, D) \mid \exists \Pi \subset \mathcal{A}(I)_{\xi}, x=r\left(\Pi_{p, 0}\right)\right\}
$$

is Zariski dense in $V(L, D)$. (Note that by our definitions of $\mathcal{A}(I)_{\xi}$ in $\S 3$ and of $f_{\infty}$, for $\Pi \subset \mathcal{A}(I)_{\xi}$, we have $\operatorname{tr} \Pi_{\infty}\left(f_{\infty}\right) \neq 0$.) By [42, Proposition 2.2], the function $\pi_{p} \longmapsto \operatorname{tr} \pi_{p}\left(f_{\tau, h}^{*}\right)$ is regular on the Bernstein variety $\mathfrak{z}_{2}$. Therefore, $\operatorname{tr} \pi_{p}\left(f_{\tau, h}^{*}\right)=0$ for any non-discrete series representation $\pi_{p}$.

This implies if $\gamma_{p}$ does not come from an element of $G\left(\mathbb{Q}_{p}\right)$, i.e., the conjugacy class of $N \delta$ does not include an element of $G\left(\mathbb{Q}_{p}\right)$, then

$$
O_{\gamma_{p}}\left(f_{\tau, h}^{*}\right)=0
$$

cf. $\left[3\right.$, Lemma 3.3]. This means that $f_{\tau, h}^{*}$ comes from a function $f_{\tau, h} \in C_{c}^{\infty}\left(G\left(\mathbb{Q}_{p}\right)\right)$, such that

$$
\operatorname{tr} \pi_{p}\left(f_{\tau, h}\right)=e(\delta) \operatorname{tr} \pi_{p}^{*}\left(f_{\tau, h}^{*}\right),
$$

where $\pi_{p} \longmapsto \pi_{p}^{*}$ is the Jacquet-Langlands correspondence between the set of smooth irreducible representations of $G\left(\mathbb{Q}_{p}\right)$ and $G^{*}\left(\mathbb{Q}_{p}\right)$. The functions $f_{\tau, h}^{*}$ and $f_{\tau, h}$ have matching orbital integrals, hence $f_{\tau, h}$ and $\phi_{\tau, h}$ have matching (twisted) orbital integrals. 
We have the following theorem. It says that the fixed points which have non-trivial contribution to the trace formula can be parameterized by Kottwitz triples, and their contribution have the usual description as in the quasi-split case of [39].

Theorem 5.5. Let $f^{p} \in C_{c}^{\infty}\left(G\left(\mathbb{A}_{f}^{p}\right)\right), h \in C_{c}^{\infty}\left(G\left(\mathbb{Z}_{p}\right)\right)$ and $\tau \in$ Frob $^{j} I_{E} \subset W_{E}$, then

$$
\operatorname{tr}\left(\tau \times h f^{p} \mid H_{\xi}\right)=\sum_{\left(\gamma_{0} ; \gamma, \delta\right)} c\left(\gamma_{0} ; \gamma, \delta\right) O_{\gamma}\left(f^{p}\right) T O_{\delta \sigma}\left(\phi_{\tau, h}\right) \operatorname{tr} \xi\left(\gamma_{0}\right),
$$

where the sum runs over degree- $j$-Kottwitz triples, and $c\left(\gamma_{0} ; \gamma, \delta\right)$ is a volume factor defined as in [25, p. 441]. The Haar measures on $G\left(\mathbb{Q}_{p}\right)$ respectively $G\left(\mathbb{Q}_{p^{t}}\right)$ are normalized by giving $G\left(\mathbb{Z}_{p}\right)$ respectively $G\left(\mathbb{Z}_{p^{t}}\right)$ volume 1.

Proof. By Proposition 5.1 we have the formula

$$
\operatorname{tr}\left(\tau \times h f^{p} \mid H_{\xi}\right)=\sum_{\varepsilon} \operatorname{vol}\left(I_{\varepsilon}(\mathbb{Q}) \backslash I_{\varepsilon}\left(\mathbb{A}_{f}\right)\right) O_{\gamma}\left(f^{p}\right) T O_{\delta \sigma}\left(\phi_{\tau, h}\right) \operatorname{tr} \xi(\varepsilon),
$$

where $\varepsilon$ runs over the set of conjugacy classes of $I(\mathbb{Q})$ such that $A_{\varepsilon}$ is non-empty. By the above vanishing theorem, only those $\varepsilon$ such that the conjugacy class of $N \delta$ for the associated $\delta$ have non-trivial contribution to the this formula, in which case we can find a $\gamma_{0} \in G(\mathbb{Q})$ such that $\left(\gamma_{0} ; \gamma, \delta\right)$ forms a Kottwitz triple for $G$. Moreover, in this case the associated group $I\left(\gamma_{0} ; \gamma, \delta\right)$ to $\left(\gamma_{0} ; \gamma, \delta\right)$ can be taken as $I_{\varepsilon}$. Fix such a Kottwitz triple $\left(\gamma_{0} ; \gamma, \delta\right)$. The number of conjugacy classes $\varepsilon$ which gives this triple equals to $\left|\operatorname{ker}\left(\operatorname{ker}^{1}\left(\mathbb{Q}, I\left(\gamma_{0} ; \gamma, \delta\right)\right) \rightarrow \operatorname{ker}^{1}(\mathbb{Q}, G)\right)\right|(\operatorname{cf}$. [29, Proposition 6.11]). Clearly the traces of $\varepsilon$ and $\gamma_{0}$ are the same for the $\overline{\mathbb{Q}}_{l}$-representation $\xi$. Then one can rewrite the above formula in the form as in the theorem.

\section{A character identity}

We continue the study of the cohomology group $H_{\xi}$. Let the notations be as in last section. Since the global reductive group $G$ has trivial endoscopic groups, by the procedure of pseudostabilization, we get the following corollary.

Corollary 6.1. For $\tau \in$ Frob $^{j} I_{E} \subset W_{E}, h_{1} \in C_{c}^{\infty}\left(O_{D_{1}}^{\times}\right), \ldots, h_{r} \in C_{c}^{\infty}\left(O_{D_{r}}^{\times}\right), h^{\prime} \in C_{c}^{\infty}\left(O_{D^{\prime}}^{\times}\right)$, $h_{0} \in C_{c}^{\infty}\left(\mathbb{Z}_{p}^{\times}\right)$, with all these functions take values in $\mathbb{Q}$ and $h^{\prime}$ invariant under conjugation, we have the following equality

$$
\operatorname{Ntr}\left(\tau \times h_{1} \times \cdots \times h_{r} \times h^{\prime} \times h_{0} \times f^{p} \mid H_{\xi}\right)=\operatorname{tr}\left(f_{\tau, h_{1}} \times \cdots \times f_{\tau, h_{r}} \times h^{\prime} \times h_{0}^{\prime} \times f^{p} \mid H_{\xi}\right),
$$

where $N=\operatorname{dim} r_{-\mu}, h_{0}^{\prime} \in C_{c}^{\infty}\left(\mathbb{Q}_{p}^{\times}\right)$has support in $p^{-t} \mathbb{Z}_{p}^{\times}$and is defined by $h_{0}^{\prime}(x)=$ $h_{0}\left(p^{t} x\right)$ for all $x \in p^{-t} \mathbb{Z}_{p}^{\times}$.

Proof. With the expression in the last theorem, we can apply Kottwitz's argument of pseudostabilization in [24] (see also the proof of [38, Corollary 9.4]). We just sketch the most important steps in the calculation. First, note the functions $\phi_{\tau, h}=\phi_{\tau, h_{1}} \times \cdots \phi_{\tau, h_{r}} \times$ $\phi_{h^{\prime}} \times h_{0}$ and $f_{\tau, h_{1}} \times \cdots \times f_{\tau, h_{r}} \times h^{\prime} \times h_{0}^{\prime}$ have matching (twisted) orbital integrals. As in $\left[24\right.$, p. 657] Kottwitz introduced a function $f_{\infty}$ on $G(\mathbb{R})$ depending on $\xi$. Consider the function

$$
f=f_{\tau, h_{1}} \times \cdots \times f_{\tau, h_{r}} \times h^{\prime} \times h_{0}^{\prime} \times f^{p} \times f_{\infty}
$$


be the function on $G(\mathbb{A})$. Then arguing as [24, pp. 661-663] we get the left hand side equals

$$
N \tau(G) \sum_{\gamma_{0}} S O_{\gamma_{0}}(f),
$$

where $\tau(G)$ is the Tamagawa number of $G, \gamma_{0}$ runs through the stable conjugacy classes in $G(\mathbb{Q})$ and $S O_{\gamma_{0}}(f)$ is the stable orbital integral. By the Arthur-Selberg trace formula for $f$ we get it equals

$$
N \sum_{\pi} m(\pi) \operatorname{tr}(f \mid \pi)
$$

where $\pi$ runs through automorphic representations for $G$ with central character $\check{\xi}$. By $[24$, Lemma 4.2] this can be rewritten as

$$
\sum_{\pi_{f}} \operatorname{tr}\left(f_{\tau, h_{1}} \times \cdots \times f_{\tau, h_{r}} \times h^{\prime} \times h_{0}^{\prime} \times f^{p} \mid \pi_{f}\right) \sum_{\pi_{\infty}} m\left(\pi_{f} \otimes \pi_{\infty}\right) e p\left(\pi_{\infty} \otimes \xi\right),
$$

where ep denotes the Euler-Poincare characteristic as in [24]. Now Matsushima's formula shows that this equals

$$
\operatorname{tr}\left(f_{\tau, h_{1}} \times \cdots \times f_{\tau, h_{r}} \times h^{\prime} \times h_{0}^{\prime} \times f^{p} \mid H_{\xi}\right) .
$$

Consider the case $r=1$. Then the above corollary combined with Theorem 3.4 gives us the following character identity.

Proposition 6.2. For $\rho \in \operatorname{Irr}\left(D^{\times}\right)$with L-parameter $\varphi_{\rho}, h \in C_{c}^{\infty}\left(O_{D}^{\times}\right)$with values in $\mathbb{Q}$ and the conjugacy class $\{\mu\}$ of cocharacters $\mu$ defined in $\xi 4$, we have the identity

$$
\operatorname{tr}\left(f_{\tau, h} \mid \rho\right)=\operatorname{tr}\left(\tau\left|\left(\left.r_{-\mu} \circ \varphi_{\rho}\right|_{W_{E}}\right)\right|-\left.\right|^{(1-n) / 2}\right) \operatorname{tr}(h \mid \rho) .
$$

Proof. As in the proof of [40, Theorem 8.1], it is equivalent to prove the equality for the corresponding quasi-EL case. With the notation of $\S 3$, we have $G_{\mathbb{Q}_{p}}=D^{\times} \times \mathbb{Q}_{p}^{\times}$. By Theorem 3.4 we know

$$
H_{\xi}=\sum_{\pi_{f}} a\left(\pi_{f}\right) \pi_{f} \otimes\left(r_{-\mu} \circ \varphi_{\pi_{p}} \mid W_{E}\right)|-|^{(1-n) / 2} .
$$

By $\left[15\right.$, Lemma 1], we can globalize $\rho$ to an irreducible admissible representation $\pi_{f}$ of $G\left(\mathbb{A}_{f}\right)$ such that there is an algebraic representation $\xi$ of $G$ with the $\pi_{f}$-isotypic component of $H_{\xi}$

$$
H\left(\pi_{f}\right):=\operatorname{Hom}_{G\left(\mathbb{A}_{f}\right)}\left(\pi_{f}, H_{\xi}\right) \neq 0 .
$$

Take some integer $m \geqslant 1$ such that $h \in C_{c}^{\infty}\left(G\left(\mathbb{Z}_{p}\right)\right)$ is bi- $K_{p}^{m}$-invariant. Also, take a compact open compact subgroup $K^{p} \subset G\left(\mathbb{A}_{f}^{p}\right)$ such that $\pi_{f}^{p}$ has $K^{p}$-invariants. Let $K=K_{p}^{m} K^{p}$, then since $H_{\xi}^{K}=\sum(-1)^{i} H^{i}\left(S h_{K, \overline{\mathbb{Q}}_{p}}, \mathcal{L}_{\xi}\right)$ is finite dimensional, there are only finitely many irreducible admissible representations $\pi_{f}^{\prime}$ with invariants under $K$ and $H\left(\pi_{f}^{\prime}\right) \neq 0$. There exists a function $f^{p} \in C_{c}^{\infty}\left(G\left(\mathbb{A}_{f}^{p}\right)\right)$ bi- $K^{p}$-invariant with $\operatorname{tr}\left(f^{p} \mid \pi_{f}^{p}\right)=1$ and such that whenever $\pi_{f}^{\prime}$ is an irreducible representation of $G\left(\mathbb{A}_{f}\right)$ with $H\left(\pi_{f}^{\prime}\right) \neq 0$, with invariants under $K$, and $\operatorname{tr}\left(f^{p} \mid\left(\pi_{f}^{\prime}\right)^{p}\right) \neq 0$, then $\pi_{f} \simeq \pi_{f}^{\prime}$. Note the $N$ of Corollary 6.1 
equals $n$ under our assumption here. Now we compute the trace of the function $f_{\tau, h} \times f^{p}$ on $H_{\xi}$ :

$$
\operatorname{tr}\left(f_{\tau, h} \times f^{p} \mid H_{\xi}\right)=n a\left(\pi_{f}\right) \operatorname{tr}\left(f_{\tau, h} \mid \pi_{p}\right) .
$$

By the above corollary

$$
n a\left(\pi_{f}\right) \operatorname{tr}\left(\tau\left|r_{-\mu} \circ \varphi_{\pi_{p}}\right| W_{E}|-|^{(1-n) / 2}\right) \operatorname{tr}\left(h \mid \pi_{p}\right)=n a\left(\pi_{f}\right) \operatorname{tr}\left(f_{\tau, h} \mid \pi_{p}\right) .
$$

We can deduce the desired identity.

\section{The cohomology of Shimura varieties II}

Now consider the general case that $1 \leqslant r \leqslant s$ are arbitrary integers. The cocharacter $\mu: \mathbb{G}_{m} \longrightarrow G_{\overline{\mathbb{Q}}_{p}}$ has a decomposition $\mu=\left(\mu_{1}, \ldots, \mu_{r}, \mu^{\prime}, \mu_{0}\right)$, which induces a decomposition of $r_{-\mu}=\left(\otimes_{i=1}^{r} r_{-\mu_{i}}\right) \otimes r_{-\mu^{\prime}} \otimes r_{-\mu_{0}}$ when restricting on $\widehat{G}$. In fact one sees easily that $r_{-\mu^{\prime}}$ is the trivial representation. $E$ is the composition of local reflex fields $F_{\varpi_{i}}$ for each $\mu_{i}$ with $1 \leqslant i \leqslant r$. Any smooth irreducible representation $\pi$ of $G\left(\mathbb{Q}_{p}\right)=\prod_{i=1}^{r} D_{i}^{\times} \times\left(D^{\prime}\right)^{\times} \times \mathbb{Q}_{p}^{\times}$has a tensor product decomposition $\pi=\bigotimes_{i=1}^{r} \pi_{i} \otimes$ $\pi^{\prime} \otimes \chi$. Accordingly we have $L$-parameters $\varphi_{\pi}, \varphi_{\pi_{1}}, \ldots, \varphi_{\pi_{r}}, \varphi_{\pi^{\prime}}, \varphi_{\chi}$. Then the properties of the test functions lead to the following character identity in the general case.

Proposition 7.1. For any irreducible representation $\pi$ of $G\left(\mathbb{Q}_{p}\right)$ with L-parameter $\varphi_{\pi}$, let $h \in C_{c}^{\infty}\left(G\left(\mathbb{Z}_{p}\right)\right)$ have the form as $h=h_{1} \times \cdots \times h_{r} \times h^{\prime} \times h_{0}$ with $h_{i} \in C_{c}^{\infty}\left(O_{D_{i}}^{\times}\right)$for $1 \leqslant i \leqslant r, h^{\prime} \in C_{c}^{\infty}\left(O_{D^{\prime}}^{\times}\right)$, and $h_{0} \in C_{c}^{\infty}\left(\mathbb{Z}_{p}^{\times}\right)$, we have the identity

$$
\operatorname{tr}\left(f_{\tau, h} \mid \pi\right)=\operatorname{tr}\left(\tau\left|\left(r_{-\mu} \circ \varphi_{\pi} \mid W_{E}\right)\right|-\left.\right|^{r(1-n) / 2}\right) \operatorname{tr}(h \mid \pi) .
$$

Proof. This comes from the decompositions of $f_{\tau, h},\left(r_{-\mu} \circ \varphi_{\pi} \mid W_{E}\right)|-|^{r(1-n) / 2}, h$ and the corresponding identities for each factor $f_{\tau, h_{i}}, 1 \leqslant i \leqslant r$, and for $h_{0}^{\prime}$.

Theorem 7.2. We have the identity

$$
H_{\xi}=\sum_{\pi_{f}} a\left(\pi_{f}\right) \pi_{f} \otimes\left(r_{-\mu} \circ \varphi_{\pi_{p}} \mid W_{E}\right)|-|^{r(1-n) / 2}
$$

as virtual $G\left(\mathbb{Z}_{p}\right) \times G\left(\mathbb{A}_{f}^{p}\right) \times W_{E}$-representations. The notations in this identity are the same as those in Theorem 3.4.

Proof. As in the proof of Corollary 6.1, we can take the pseudostabilization of the formula in Theorem 5.4 to get the equality

$$
\operatorname{tr}\left(\tau \times h \times f^{p} \mid H_{\xi}\right)=N^{-1} \operatorname{tr}\left(f_{\tau, h} f^{p} \mid H_{\xi}\right) .
$$

On the other hand, Matsushima's formula implies

$$
H_{\xi}=N \sum_{\pi_{f}} a\left(\pi_{f}\right) \pi_{f} .
$$

Put this into the above formula and take account the equality of Proposition 7.1, we get

$$
\operatorname{tr}\left(\tau \times h \times f^{p} \mid H_{\xi}\right)=\sum_{\pi_{f}} a\left(\pi_{f}\right) \operatorname{tr}\left(\tau\left|\left(r_{-\mu} \circ \varphi_{\pi_{p}}\right)\right|-\left.\right|^{r(1-n) / 2}\right) \operatorname{tr}\left(h f^{p} \mid \pi_{f}\right) .
$$

This gives the desired identity. 
As in the proof of the theorem, the crucial point is that we have the character identity of Proposition 6.2, which in turn needs first Theorem 5.4 to hold true to have the definitions of the functions $f_{\tau, h}$. This identity plus that in [40, Theorem 8.1] can be used to prove new cases for the description of cohomology of Shimura varieties. For example, the compact unitary Shimura varieties with trivial endoscopy, such that the local reductive groups at $p$ are products of (Weil restrictions of) $D^{\times}$and $G L_{n}$ where $D$ is a central division algebra over a finite extension of $\mathbb{Q}_{p}$ with invariant $\frac{1}{n}$. Moreover, to apply the results in this paper, we have to require the local cocharacters for the factors $D^{\times}$are the same as those studied here. The case of $n=2$ in [24] but for arbitrary $p$ (with the above requirement on local cocharacters at ramified places) will be a typical example. We will treat this case and the related quaternionic Shimura varieties in [41].

From this theorem we get the following corollary concerning the local semisimple Hasse-Weil zeta functions of our Shimura varieties. For the definition of local semisimple Hasse-Weil zeta functions and local semisimple automorphic $L$ functions, see [32]. Let $\widetilde{E}$ be the global reflex field, and $v$ be a place of $\widetilde{E}$ above $p$ such that $E=\widetilde{E}_{v}$.

Corollary 7.3. In the situation of the theorem, let $K \subset G\left(\mathbb{A}_{f}\right)$ be any sufficiently small open compact subgroup. Then the semisimple local Hasse-Weil zeta function of $S h_{K}$ at the place $v$ of $\widetilde{E}$ is given by

$$
\zeta_{v}^{s s}\left(S h_{K}, s\right)=\prod_{\pi_{f}} L^{s s}\left(s-r(n-1) / 2, \pi_{p}, r_{-\mu}\right)^{a\left(\pi_{f}\right) \operatorname{dim} \pi_{f}^{K}} .
$$

Proof. We can assume that $K$ has the form as $K^{p} K_{p} \subset G\left(\mathbb{A}_{f}^{p}\right) \times G\left(\mathbb{Z}_{p}\right)$. Then the corollary follows from the previous theorem and the definitions.

In the case $r=1$ Dat has proved the Weight-Monodromy conjecture for these Shimura varieties (cf. $[8,5.2])$. In fact, there the varieties involved are of the form $\mathcal{M}_{D r, m} / \Gamma$ where $\Gamma \subset G L_{n}(E)$ is some torsion free discrete subgroup. Here our Shimura varieties have the same connected components as $\mathcal{M}_{D r, m} / \Gamma$ for suitable $\Gamma$. By $[32, \S 2]$ one can recover the classical Hasse-Weil zeta function.

Corollary 7.4. Let $r=1$ and $K \subset G\left(\mathbb{A}_{f}\right)$ be any sufficiently small open compact subgroup in the situation of the theorem. Then the local Hasse-Weil zeta function of $S h_{K}$ at the place $v$ of $\widetilde{E}$ is given by

$$
\zeta_{\nu}\left(S h_{K}, s\right)=\prod_{\pi_{f}} L\left(s-r(n-1) / 2, \pi_{p}, r_{-\mu}\right)^{a\left(\pi_{f}\right) \operatorname{dim} \pi_{f}^{K}} .
$$

Finally we remark that, assuming the results of [28], in the above corollary there will be no restriction for the integer $r$, i.e., it can be an arbitrary positive integer, see the paragraph under Corollary 1.3 in the introduction.

Acknowledgements. I would like to thank Peter Scholze sincerely, since without his encouragement and suggestions this work would not be finished. I want to thank Yoichi Mieda and Michael Rapoport for their useful remarks. I should also thank the referee 
for careful reading and valuable suggestions. This work started while the author was a postdoc at the Mathematical Institute of the University of Bonn, and was finished after the author moved to the University of Regensburg. This work was supported by the SFB/TR 45 'Periods, Moduli Spaces and Arithmetic of Algebraic Varieties' and the SFB 1085 'Higher Invariants' of the DFG.

\section{References}

1. J. Arthur and L. Clozel, Simple Algebras, Base Change, and the Advanced Theory of the Trace Formula, Annals of Mathematics Studies, 120 (Princetion University Press, 1989).

2. A.-M. Aubert, P. Baum, R. Plymen and M. Solleveld, The local Langlands correspondence for inner forms of $S L_{n}$, preprint, 2013, arXiv:1305.2638.

3. A.-I. Badulescu, Un résultat de transfert et un résultat d'intégrabilité locale des caractères en caractéristique non nulle, J. Reine Angew. Math. 565 (2003), 101-124.

4. J.-F. Boutot and H. Carayol, Uniformisation $p$-adique des courbes de Shimura: Les Théorèmes de Čerednik et de Drinfeld, in Courbes Modulaires et Courbes de Shimura, Astérisque, 196-197, pp. 45-158 (1991).

5. J.-F. Boutot And T. Zink, The $p$-adic uniformization of Shimura curves, preprint 95-107, Universität Bielefeld (1995), available at https://www.math.uni-bielefeld.de/ $\sim_{\mathrm{z}}$ ink/p-adicuni.ps.

6. P. Boyer, Monodromie du faisceau pervers des cycles évanescents de quelques variétés de Shimura simples, Invent. Math. 177 (2009), 239-280.

7. H. Carayol, Non-abelian Lubin-Tate theory, in Automorphic Forms, Shimura varieties, and L-Functions, Volume 2, Perspectives in Mathematics, 11, pp. 15-39 (Academic Press, 1990).

8. J.-F. DAT, Théorie de Lubin-Tate non-abélienne et représentations elliptiques, Invent. Math. 169(1) (2007), 75-152.

9. P. Deligne, D. Kazhdan and M.-F. Vignéras, Représentations des algèbres centrales simples p-adiques, in Représentations des groupes réductifs sur un corps local, pp. 33-117 (Hermann, Paris, 1984).

10. V. G. Drinfeld, Coverings of p-adic symmetric domains, Funct. Anal. Appl. 10 (1976), $107-115$.

11. G. Faltings, A relation between two moduli spaces studied by V. G. Drinfeld, in Algebraic Number Theory and Algebraic Geometry, Contemporary Mathematics, 300, pp. 115-129 (American Mathematical Society, Providence, RI, 2002).

12. L. Fargues, Cohomologie des espaces de modules de groupes p-divisibles et correspondances de Langlands locales, in Variétés de Shimura, espaces de Rapoport-Zink de correspondances de Langlands locales, Astérisque, 291, pp. 1-199 (2004).

13. L. FArgues, L'isomorphisme entre les tours de Lubin-Tate et de Drinfeld et applications cohomologiques, in L'isomorphisme entre les tours de Lubin-Tate et de Drinfeld, Progress in Mathematics, 262, pp. 1-325 (Birkhäuser, Basel, 2008).

14. T. J. HAines, The stable Bernstein center and test functions for Shimura varieties, in Automorphic Forms and Galois Representations, Volume 2, London Mathematical Society Lecture Note Series, 415, pp. 118-186 (Cambridge University Press, 2014).

15. M. HARRIS, Supercuspidal representations in the cohomology of Drinfel'd upper half spaces; elaboration of Carayol's program, Invent. Math. 129 (1997), 75-119.

16. M. HARris, The local Langlands conjecture for $G L(n)$ over a $p$-adic field, $n<p$, Invent. Math. 134(1) (1998), 177-210. 
17. M. Harris, Galois properties of cohomological automorphic forms on $G L(n), J$. Math. Kyoto Univ. 39(1) (1999), 299-318.

18. M. Harris And R. TAYlor, The Geometry and Cohomology of Some Simple Shimura Varieties (Princeton University Press, 2001).

19. K. Hiraga and H. Saito, On $L$-packets for inner forms of $S L_{n}, M e m$. Amer. Math. Soc. 1013215 (2012).

20. T. Iто, Weight-monodromy conjecture for $p$-adically uniformized varieties, Invent. Math. 159(3) (2005), 607-656.

21. R. E. Kоттwitz, Rational conjugacy classes in reductive groups, Duke Math. J. 49(4) (1982), 785-806.

22. R. E. Коттwitz, Shimura varieties and twisted orbital integrals, Math. Ann. 269 (1984), 287-300.

23. R. E. Коттwitz, Shimura varieties and $\lambda$-adic representations, in Automorphic Forms, Shimura Varieties, and L-functions, Volume 1, Perspectives in Mathematics, 10, pp. 161-209 (Academic Press, 1990).

24. R. E. Коттwitz, On the $\lambda$-adic representations associated to some simple Shimura varieties, Invent. Math. 108(3) (1992), 653-665.

25. R. E. Коттwitz, Points on some Shimura varieties over finite fields, J. Amer. Math. Soc. 5 (1992), 373-444.

26. R. E. Kоттwitz, Isocrystals with additional structure II, Compo. Math. 109(3) (1997), 255-339.

27. E. Mantovan, On the cohomology of certain PEL-type Shimura varieties, Duke Math. 129 (2005), 573-610.

28. Y. MiedA, Note on weight-monodromy conjecture for $p$-adically uniformized varieties, preprint, 2014, arXiv:1411.5959.

29. J. S. Milne, The points on a Shimura variety modulo a prime of good reduction, in The Zeta Functions of Picard Modular Surfaces (ed. R. P. LANGLANDS AND D. RAMAKRISHNAN), pp. 151-253 (CRM, 1992).

30. S. Morel, On the Cohomology of Certain Non-compact Shimura Varieties, Annals of Mathematics Studies, 173 (Princeton University Press, 2010).

31. M. Rapoport, On the local zeta function of quaternionic Shimura varieties with bad reduction, Math. Ann. 279 (1988), 673-697.

32. M. RApoport, On the bad reduction of Shimura varieties, in Automorphic Forms, Shimura varieties, and L-Functions, Volume 2, Perspectives in Mathematics, 11, pp. 77-160 (Academic Press, 1990).

33. M. RApOport, A guide to reduction modulo $p$ of Shimura varieties, Astérisique 298 (2005), 271-318.

34. M. RApOPORT And T. ZInk, Period Spaces for p-divisible Groups, Annals of Mathematics Studies, 141 (Princetion University Press, 1996).

35. H. Reimann, The Semi-simple Zeta Function of Quaternionic Shimura Varieties, Lecture Notes in Mathematics, 1657 (Springer, Berlin, 1997).

36. J. Rogawski, Representations of $G L(n)$ and division algebras over a $p$-adic field, Duke Math. J. 50 (1983), 161-169.

37. P. Scholze, The Langlands-Kottwitz approach for some simple Shimura varieties, Invent. Math. 192(3) (2013), 627-661.

38. P. Scholze, The local Langlands correspondence for $G L_{n}$ over $p$-adic fields, Invent. Math. 192(3) (2013), 663-715.

39. P. Scholze, The Langlands-Kottwitz method and deformation spaces of $p$-divisible groups, J. Amer. Math. Soc. 26(1) (2013), 227-259.

40. P. Scholze And S.-W. Shin, On the cohomology of compact unitary group Shimura varieties at ramified split places, J. Amer. Math. Soc. 26(1) (2013), 261-294. 
41. X. Shen, On the cohomology of some simple Shimura varieties with bad reduction, preprint, 2014, arXiv:1411.0245.

42. S.-W. Shin, On the cohomology of Rapoport-Zink spaces of EL type, Amer. J. Math. 134(2) (2012), 407-452.

43. Y. VARShavsky, P-adic uniformization of unitary Shimura varieties, Publ. Math. Inst. Hautes Études Sci. 87 (1998), 57-119.

44. Y. Varshavsky, $P$-adic uniformization of unitary Shimura varieties II, J. Differential Geom. 49(1) (1998), 75-113.

45. Y. VARShAVSKY, Lefschetz-Verdier trace formula and a generalization of a theorem of Fujiwara, Geom. Funct. Anal. 17(1) (2007), 271-319.

46. A. Zelevinsky, Induced representation of reductive $p$-adic groups. II. On irreducible representations of GL(n), Ann. Sci. Éc. Norm. Supér. (4) 13(2) (1980), 165-210. 